\title{
Is There a Motherhood Penalty in Academia? The Gendered Effect of Children on Academic Publications in German Sociology
}

\section{Mark Lutter ${ }^{1, *, \dagger}$ and Martin Schröder ${ }^{2, \dagger}$}

\author{
${ }^{1}$ Department of Sociology, University of Wuppertal, Gaußstr. 20, Wuppertal 42119, Germany and \\ ${ }^{2}$ Department of Sociology, University of Marburg, Ketzerbach 11, Marburg 35037, Germany \\ *Corresponding author. Email: lutter@uni-wuppertal.de \\ ${ }^{\dagger}$ Author names are listed alphabetically. Both authors contributed equally.
}

Submitted January 2019; revised October 2019; accepted October 2019

\begin{abstract}
Based on data that tracks curriculum vitae (CV) and publication records as well as survey information from sociologists in German academia, we examine the effects of parenthood on the publication output of male and female academics that were present in German universities or research institutes in the year 2013. Results indicate that having children leads to a significant decline in the number of publications by women on average, while not affecting the number of publications by men. However, the gendered effect of children on productivity hardly mitigates differences in publication output between men and women, as women still publish about 20 per cent less than men after controlling for the adverse effects of children on productivity. The gendered effect of childbearing depends partly on prior levels of women's academic achievements, suggesting a mechanism of performance-driven self-selection. Lower-performing women tend to suffer a stronger motherhood penalty than better performing women, while the publication output of successful women (who have been granted academic awards) is not reduced through childbirth. The results indicate that women are better at managing the 'double burden' of kids and career if external, award-giving committees have bestowed prestige upon them or indicated their potential for a scientific career.
\end{abstract}

\section{Introduction}

Across disciplines, age groups, and cohorts, men in academia publish significantly more than women (Cole and Zuckerman, 1987: p. 119; Cole and Singer, 1991; Xie and Shauman, 1998, 2003; Sax et al., 2002: p. 424; Stack, 2004; Leahey, 2006: p. 756; Lutter and Schröder, 2016). What explains this gender gap in publication output? Empirical studies suggest that raising children is generally one of the main factors explaining women's disadvantaged labor market position (Correll, Benard and Paik, 2007; Petit, 2007: p. 385; Sigle-Rushton and Waldfogel, 2007: pp. 67-77). The same is true in academia: scholars attribute the publication gap to motherhood and childcare responsibilities (Mason, Wolfinger and Goulden, 2013: p. 29; Rivera, 2017: p. 1114).

However, empirical findings on whether motherhood actually explains the publication gap in academia show mixed results (see the reviews in Sax et al., 2002: p. 425; Stack, 2004: p. 893; Hunter and Leahey, 2010: p. 433; Joecks, Pull and Backes-Gellner, 2014: p. 517). Some 
early studies, which led to long-standing hypotheses, indeed confirm that motherhood decreases the publication output of women (Hargens, McCann and Reskin, 1978: p. 161; Long, 1990); yet others argue that only young children decrease publication output (Kyvik, 1990: p. 158; Kyvik and Teigen, 1996: p. 69; Stack, 2004: p. 914; Mason, Wolfinger and Goulden, 2013: p. 29). Some do not find any effect. Hamovitch and Morgenstern (1977: p. 643) were the first to conclude that, after controlling for academic rank, teaching duties, years since $\mathrm{PhD}$ completion, type of university, and academic discipline, 'the remaining sex difference in publication (20 per cent fewer articles published by women than by men) is not due to any relationship or tradeoff between children and productivity'.

In line with Hamovitch and Morgenstern, newer studies also do not find that children affect the publication output of women differently than that of men (Cole and Zuckerman, 1987: p. 125; Sax et al., 2002: p. 435; Fox, 2005: p. 146; Jaksztat, 2017: p. 357). For example, Sax et al. (2002: p. 438) find that 'family-related factors do not interfere with scholarly productivity'. Krapf, Ursprung and Zimmermann (2017) find no general effect in a large sample of economists but suggest that there might be a motherhood penalty for very young mothers or those with two or more children. They also find that fathers increase their research productivity after their first child. Some studies even find that children increase the publication output of mothers (Toutkoushian and Bellas, 1999; Nakhaie, 2002; Stack, 2004: p. 913; Joecks, Pull and Backes-Gellner, 2014: p. 526).

These differing results may be due to the time period, country, and disciplines that each study focuses on. However, we suggest that it is also important to distinguish between-level effects from within-level effects. Notably, many prior studies base their conclusions solely on cross-sectional or between-level effects. These studies, therefore, compare groups but do not take into account individual career changes, i.e. within-level effects, obtained through fixed-effects regressions.

Second, we suggest to control for possible selection effects before childbirth, as researchers who have been assured of their academic potential early on may tenaciously stick to an academic career and continue to publish even after having children (Cole and Zuckerman, 1987: p. 125; Fox, 2005: p. 145; Joecks, Pull and BackesGellner, 2014: p. 520). For researchers who have not been assured of their academic potential and, thus, may feel a stronger incommensurability of their role as researcher with other parts of their social identity, parenthood may not increase their determination to publish but instead provide a road out of academia (Haas, Koeszegi and Zedlacher, 2016; Haas and Koeszegi, 2017).

In this study, we therefore analyze how having children affects the publication rate of men and women differently while controlling for prior academic performance as an indication for possible selection effects. We also disentangle between-level effects from within-level effects and test how parenthood affects publication output differently if we distinguish between low and high levels of prior academic performance.

In doing so, we use a unique panel dataset that tracks $\mathrm{CV}$ and publication records from virtually all sociologists working in all sociology departments in Germany in the year 2013. We combine these data with an online survey, in which we asked all sociologists whether they have children, and if so, when they were born. We study German sociologists before tenure to assure that everyone in our dataset is under similar institutionalized pressure to publish while deciding about parenthood. In contrast to countries such as France, the United Kingdom, or the United States, Germany has virtually no permanent positions below a full or associate professorship and no established tenure track system. In addition, German labor law (the so-called 'Wissenschaftszeitvertragsgesetz') forces everyone to leave academia after 12 years of temporary employment, making it a drastic 'up or out' system. Every childbirth prolongs the period, during which one can legally stay in academia, by 2 years. Scholars, therefore, have to publish as much as they can during the typical age for parenthood (the average age for getting a tenured professorship is forty-one in Germany, see Destatis, 2018: p. 279).

We focus on the discipline of sociology because of its relatively equal representation of men and women (unlike the natural sciences), making it ideally suited to analyze career trajectories of both genders (Stack, 1994: p. 81, 2002: p. 286; Leahey, 2006: p. 760; JungbauerGans and Gross, 2013). Sociology is uniquely suited to study the impact of children on publication output because unlike the lab sciences, work in sociology is relatively portable. It is easier to write an essay or conduct statistical analyses from home than to conduct an experiment. Because of both relatively equal gender representation and more portable work, researchers suggest that if children matter in fields such as sociology, they are likely to matter even more in the natural sciences (Hunter and Leahey, 2010: p. 436f.; Mason, Wolfinger and Goulden, 2013: p. 25).

Since our survey as well as publication and CV data tracks information at one point in time, we can only observe those currently in the system. This survivorship effect may bias our results, as women are more likely than 
men to drop out of academia, especially after childbirth. However, as we analyze regression models separately for women and men, our analytic strategy builds on intra-gender comparisons from retrospective data. This enables us to analyze differences in academic careers comparing women to women and men to men. This does not rule out survivorship effects completely, but at least treats groups with different dropout probabilities separately.

\section{The gendered effect of children on publication output: theoretical considerations and empirical results}

Several mechanisms may explain why and in what direction children affect academic research output (for a review of these mechanisms, see Cole and Zuckerman, 1987; Stack, 2004; Fox, 2005; Hunter and Leahey, 2010; Joecks, Pull and Backes-Gellner, 2014). In line with Joecks, Pull and Backes-Gellner (2014), children may influence publication output through (i) effects of self-selection, (ii) effects of time constraints, and (iii) incentive effects as well as changes in preferences.

The first mechanism, self-selection, may operate in two opposed ways, depending on career orientation and how much academic acclaim researchers received during early career stages. On the one side, self-selection may drive the most determined, career-oriented academics towards parenthood, as more productive researchers may assume that they can keep up their publication output after having children. The measurable effect of children on productivity could then be neutral or positive. This has been alluded to in the literature but not systematically tested (Stack, 2004: p. 899). For example, Cole and Zuckerman (1987: p. 125) find no effect of children on the research productivity of US female scientists in various disciplines, suggesting this is because childbearing women in their sample have 'eliminate[d] almost everything but work and family' from their lives to remain productive. The proposed mechanism is that researchers who are more assured that investment into academia will 'pay off' remain committed to their career after becoming parents, so that the most productive researchers experience only a small decline in publication output after childbirth.

On the other side, researchers who have been less assured of their academic success may decide to drop out of academic publishing when faced with the 'double burden' of parenthood and career. We should, thus, see that having children interacts with prior signals of success. In societies with traditional gender roles, such as
Germany (Blossfeld, Drobnic and Rohwer, 2001; Schulz and Blossfeld, 2006; Gangl and Ziefle, 2015; Sieverding et al., 2018), this should be more the case for women than men. When their partner is in charge of most housework, men do not need to be assured of their eventual academic success to keep on publishing after having children, since the traditional separation of household labor can even help them to increase their publications. Recent research suggests that male academics indeed benefit from traditional gender roles, using parental leave to continue publishing (Antecol, Bedard and Stearns, 2018).

The second mechanism is that children can lower publication productivity by consuming time that is otherwise available for research. This effect is directly related to the first mechanism and may again be related to prior signals of academic success. If academics have been assured of their potential, they may continue to publish post-childbirth, for example by placing their children in professional childcare. This mechanism therefore also suggests that researchers with lower early signals of academic achievement experience a stronger decline in publication output after childbirth.

The third mechanism is that children may change work incentives and preferences-again depending on signals received before parenthood. On the one hand, when academics received signals that assure them of a realistic chance for an academic career, then parenthood may increase incentives to seek the economic security of tenure, which in turn requires publications. Thus, children may incentivize parents to publish more, but only if researchers adopted a breadwinner role and can realistically assume to fulfil this role by getting a professorship.

Parenthood also provides an alternative source of identity (see Gangl and Ziefle, 2015) and can lead to severe stress, especially for mothers (Giesselmann, Hagen and Schunck, 2018). Publication output may therefore decline after childbirth. When researchers have not been encouraged before childbirth, through early success or the signalling function of awards, they may abandon efforts to publish when an alternative source of identification arises or stress increases. However, those whose identity as a researcher have been confirmed through academic success may be more willing to pursue research after childbirth.

Empirical results also suggest that the effect of children on post-birth productivity depends on prior levels of academic success. Fox (2005: p. 146) finds that 'young children are not associated with depressed publication productivity' but cautions that this is true for 'women who have survived a rigorous and demanding process of scrutiny, selection, and evaluation in science', 
suggesting that the effect of children interacts with prior signals of academic achievement. Hunter and Leahey (2010: p. 447) surveyed linguists and sociologists in US research universities and show that parents have more publications at each career step than childless researchers. However, their estimations also suggest that researchers with children experience a drop in publications relative to their own pre-birth productivity. This suggests that, on the one hand, children depress the publication productivity trajectory of each researcher. On the other hand, it suggests that only the most determined researchers have children in the first place, which may overshadow the negative effect of children on individual productivity. Group-comparisons may, therefore, be problematic to estimate the effect of children on the individual productivity of researchers.

\section{Data and Methods}

\section{Data}

We use a unique panel dataset that covers the pre-tenure careers of all sociologists with at least one publication, who were employed at a sociology department in a German university in 2013. To collect the data, we identified all seventy-five sociology departments in Germany, plus two research institutes. Based on department and faculty websites, we hand-coded all available CV data and publications. The constructed longitudinal dataset contains individual time-series data of virtually all German academic sociologists, starting from their first publication until tenure as a full or associate professor or the year 2013. We limit our data to sociologists who obtained their PhD after the year 1980. After collecting this data, we conducted an email survey, in which we asked all academics in our database whether or not they have children and, if so, when these were born. The survey's response rate was 64 per cent. ${ }^{1}$ After strictly anonymizing all information, we integrated both data sources and arranged them as a person-year panel. We collected data on 1,260 sociologists, nested in 11,833 person-years. The regressions below use the subset of those 64 per cent who responded to our survey and thus had all necessary variables. The final dataset contains time-varying profiles with 7,667 person-year observations from 805 sociologists. Our dependent variable (DV) is the annual number of co-author adjusted peer-reviewed Social Science Citations Index (SSCI) journal articles (see next section for details). We lag all independent variables by 1 year to avoid simultaneity bias and distinguish current and prior publications. This reduces the dataset to 6,846 person-year observations from 729 sociologists in the regressions below (297 women and 432 men).

\section{Dependent Variable}

Analogous to existing research, our dependent variable is the annual number of co-author adjusted articles in peerreviewed journals (cf. Fox, 2005: p. 134; Hunter and Leahey, 2010: p. 438; Joecks, Pull and Backes-Gellner, 2014: p. 523). We only consider journals listed in the SSCI because these adhere to standards of quality and peer review (Bohannon, 2013). The double-blind peer review of these journals is important for our purpose, as studies show how the work of female scientists is devalued when their gender is known (Wennerås and Wold, 1997; van den Brink and Benschop, 2011). We adjust publications for co-authorship through the formula $2 /$ (number of authors +1 ), which weighs single-authored publications as 1 publication, publications with one co-author as 0.67 , with three authors as 0.5 , and so on. In robustness tests, we also used unweighted adjustments of co-authorships (with the formula publication/number of authors) and we used no authorship adjustment (counting every publication as 1 , regardless of co-authors). Neither produced large differences compared with the main results below (available upon request). Since publication output follows Lotka's law (Lotka, 1926), the distribution of the dependent variable is non-normal and skewed. To account for this, we estimated models using a logged dependent variable. Our results are robust to these different specifications of the dependent variable (see the discussion in section 'robustness checks' and Models 3-6 in Table A1).

\section{Independent Variables}

Our main predictors are number of children (varying annually) and gender (coded as $1=$ female). Since we lag the number of children and all other independent variables by 1 year, we estimate the effect of childbearing on publications of the following years. As a robustness check, we also tested a 2-year lag for the number of children (Tables A1 and A2).

To control for how prior signals of academic achievement influence the effect of childbirth on publications, we include several variables that measure achievement and status within an academic career. These controls can be divided into three groups. The first consists of three variables that capture research experience below the level of a tenured professorship, allowing to compare researchers at similar career stages. Doctorate is a dummy variable that turns from 0 to 1 when a person obtains a doctorate degree. The dummy variable habilitation does the same when a person 
finishes a habilitation, which in Germany is like a second dissertation that scholars complete to qualify for a professorship. The third variable, years in academia, measures academic age, notably years since a researcher's first publication.

The second group of variables measures research output and academic performance to control current publications for earlier ones. The first three variables measure different types of prior publications. SSCI journal articles is the cumulated number of journal articles published until each year of a person's career. Books consists of all published monographs and textbooks, cumulated the same way. Book chapters does the same for articles published in edited volumes. Awards is the total number of academic awards a researcher received up until each time point, including all academic awards mentioned on a CV, such as best-paper awards, dissertation awards, and so on, but excluding mere stipends or research grants. Co-authors cumulates the total number of people a person has published with until each given year (including repeated co-authorships, as this measures not only show how wide the network is, but also how dense). Existing research has shown that women single-author more articles than men (Boschini and Sjögren, 2007). Since the number of co-authors may contribute to one's social network, it is a variable to take into account. International publications represents a person's number of English-language publications. German National Research Foundation (DFG) grants is the number of years during which a researcher received funding through a main research grant (Sachbeibilfe) from the DFG. Grants are essentially a resource input, so we expect them to increase future publications. ${ }^{2}$ Controlling for these variables also controls for women being disadvantaged, as they may have less access to publishing avenues and third-party funding (Wennerås and Wold, 1997; van den Brink and Benschop, 2011). Generally, we control for prior publications and research funding to compare researchers with similar prior input and output.

Note, however, that endogeneity bias cannot be completely ruled out, as academic awards are not only an input but also an outcome, which may measure potential, encouragement and even social capital that may not only lead to but also result from publications. Research grants can also both be seen as a research input and output. Strictly speaking, our results rely on seeing awards and DFG grants as inputs, and causal interpretations hinge on seeing this as plausible.

The third group of variables controls for academic mobility, measuring international and national experience with different institutions and academic cultures, which may increase publications. The first of these measures, months abroad, represents the number of months a person stayed abroad at another institution. If the CV did not contain the exact number of months (such as academic year 2012-2013 or fall term 2012/ 13), then we counted five months for a semester and ten months for an academic year. Mobility consists of the number of times an academic changed university or institution for a new academic job. Interim professor is the number of times a person worked as an interim professor ('Vertretungsprofessur' in German), a position where a non-tenured postdoc substitutes for a full professor for a limited period, usually six to twenty-four months. These variables assess performance up until each time point, so taking them into account allows comparing the effect of parenthood on productivity relative to and irrespective of a researcher's general productivity.

\section{Analytical Strategy}

Table 1 presents a descriptive overview, followed by three types of regression models: Table 2 shows random-effects models, which estimate how gender and children are related to publications before and after controlling career stages and prior accomplishment. Table 3 uses fixed-effects models, estimating how children affect the publications of a statistically typical man and woman. Rather than comparing groups (e.g. parents vs. non-parents), these models show whether the same person publishes more before or after having children. All models include the main predictor variable - number of children - and then control for selection effects by gradually including measures of academic career status and prior performance. Table 4 estimates interaction effects between performance measures and number of children for men and women. This tests whether having children affects men and women differently when they have accumulated more or less measures of academic achievement before parenthood. All models estimate cluster-robust standard errors. All independent variables except dummies are logged, to account for nonlinearity and diminishing marginal effects. For variables with zero as the minimum value, we added the constant 1 before taking the natural logarithm.

\section{Results}

\section{Descriptive Results}

Table 1 shows the means and standard deviations of all variables used in this study. Column 1 displays the statistics for our entire sample. Columns 2 and 3 separate 
Table 1. Descriptive overview on all variables: overall, by gender, and by gender and having children

\begin{tabular}{|c|c|c|c|c|c|c|c|}
\hline & $\begin{array}{l}(1) \\
\text { Overall }\end{array}$ & $\begin{array}{l}(2) \\
\text { Men }\end{array}$ & $\begin{array}{l}(3) \\
\text { Women }\end{array}$ & $\begin{array}{l}(4) \\
\text { Childless men }\end{array}$ & $\begin{array}{l}\text { (5) } \\
\text { Childless women }\end{array}$ & $\begin{array}{l}(6) \\
\text { Fathers }\end{array}$ & $\begin{array}{l}\text { (7) } \\
\text { Mothers }\end{array}$ \\
\hline $\begin{array}{l}\text { SSCI journal articles per } \\
\quad \text { year (dependent variable) }\end{array}$ & $\begin{array}{c}0.161 \\
(0.437)\end{array}$ & $\begin{array}{c}0.185 \\
(0.473)\end{array}$ & $\begin{array}{c}0.120 \\
(0.368)\end{array}$ & $\begin{array}{c}0.172 \\
(0.461)\end{array}$ & $\begin{array}{c}0.112 \\
(0.362)\end{array}$ & $\begin{array}{c}0.205 \\
(0.490)\end{array}$ & $\begin{array}{c}0.142 \\
(0.384)\end{array}$ \\
\hline Female & $\begin{array}{c}0.383 \\
(0.486)\end{array}$ & & & & & & \\
\hline Number of children & $\begin{array}{c}0.565 \\
(0.869)\end{array}$ & $\begin{array}{c}0.650 \\
(0.925)\end{array}$ & $\begin{array}{c}0.429 \\
(0.751)\end{array}$ & & & $\begin{array}{c}1.642 \\
(0.731)\end{array}$ & $\begin{array}{c}1.488 \\
(0.617)\end{array}$ \\
\hline Doctorate & $\begin{array}{c}0.459 \\
(0.498)\end{array}$ & $\begin{array}{c}0.481 \\
(0.500)\end{array}$ & $\begin{array}{c}0.422 \\
(0.494)\end{array}$ & $\begin{array}{c}0.345 \\
(0.475)\end{array}$ & $\begin{array}{c}0.310 \\
(0.463)\end{array}$ & $\begin{array}{c}0.690 \\
(0.463)\end{array}$ & $\begin{array}{c}0.697 \\
(0.460)\end{array}$ \\
\hline Habilitation & $\begin{array}{c}0.0819 \\
(0.274)\end{array}$ & $\begin{array}{c}0.0984 \\
(0.298)\end{array}$ & $\begin{array}{c}0.0552 \\
(0.228)\end{array}$ & $\begin{array}{c}0.0496 \\
(0.217)\end{array}$ & $\begin{array}{c}0.0369 \\
(0.189)\end{array}$ & $\begin{array}{c}0.173 \\
(0.378)\end{array}$ & $\begin{array}{c}0.101 \\
(0.301)\end{array}$ \\
\hline Years in academia & $\begin{array}{c}6.673 \\
(5.941)\end{array}$ & $\begin{array}{c}6.921 \\
(6.039)\end{array}$ & $\begin{array}{c}6.273 \\
(5.758)\end{array}$ & $\begin{array}{c}5.277 \\
(5.240)\end{array}$ & $\begin{array}{c}5.067 \\
(4.990)\end{array}$ & $\begin{array}{c}9.432 \\
(6.311)\end{array}$ & $\begin{array}{c}9.256 \\
(6.411)\end{array}$ \\
\hline SSCI journal articles & $\begin{array}{c}0.951 \\
(1.938)\end{array}$ & $\begin{array}{c}1.152 \\
(2.197)\end{array}$ & $\begin{array}{c}0.627 \\
(1.366)\end{array}$ & $\begin{array}{c}0.804 \\
(1.671)\end{array}$ & $\begin{array}{c}0.497 \\
(1.234)\end{array}$ & $\begin{array}{c}1.683 \\
(2.732)\end{array}$ & $\begin{array}{c}0.949 \\
(1.605)\end{array}$ \\
\hline Books & $\begin{array}{c}1.010 \\
(1.237)\end{array}$ & $\begin{array}{c}1.141 \\
(1.363)\end{array}$ & $\begin{array}{c}0.800 \\
(0.967)\end{array}$ & $\begin{array}{c}0.777 \\
(1.078)\end{array}$ & $\begin{array}{c}0.678 \\
(0.941)\end{array}$ & $\begin{array}{c}1.696 \\
(1.552)\end{array}$ & $\begin{array}{c}1.103 \\
(0.964)\end{array}$ \\
\hline Book chapters & $\begin{array}{c}4.926 \\
(7.666)\end{array}$ & $\begin{array}{c}5.490 \\
(8.368)\end{array}$ & $\begin{array}{c}4.016 \\
(6.268)\end{array}$ & $\begin{array}{c}3.843 \\
(6.715)\end{array}$ & $\begin{array}{c}3.141 \\
(5.713)\end{array}$ & $\begin{array}{c}8.006 \\
(9.882)\end{array}$ & $\begin{array}{c}6.178 \\
(7.014)\end{array}$ \\
\hline Awards & $\begin{array}{c}0.119 \\
(0.476)\end{array}$ & $\begin{array}{c}0.136 \\
(0.539)\end{array}$ & $\begin{array}{c}0.0921 \\
(0.352)\end{array}$ & $\begin{array}{c}0.109 \\
(0.458)\end{array}$ & $\begin{array}{c}0.0824 \\
(0.343)\end{array}$ & $\begin{array}{c}0.176 \\
(0.641)\end{array}$ & $\begin{array}{c}0.116 \\
(0.372)\end{array}$ \\
\hline Co-authors & $\begin{array}{c}10.70 \\
(20.79)\end{array}$ & $\begin{array}{c}11.97 \\
(23.65)\end{array}$ & $\begin{array}{r}8.657 \\
(14.84)\end{array}$ & $\begin{array}{r}8.928 \\
(17.64)\end{array}$ & $\begin{array}{c}7.354 \\
(13.98)\end{array}$ & $\begin{array}{c}16.62 \\
(30.05)\end{array}$ & $\begin{array}{c}11.88 \\
(16.36)\end{array}$ \\
\hline International publications & $\begin{array}{c}2.842 \\
(6.346)\end{array}$ & $\begin{array}{c}3.014 \\
(6.653)\end{array}$ & $\begin{array}{c}2.565 \\
(5.805)\end{array}$ & $\begin{array}{c}1.911 \\
(4.026)\end{array}$ & $\begin{array}{c}1.832 \\
(4.011)\end{array}$ & $\begin{array}{c}4.699 \\
(9.081)\end{array}$ & $\begin{array}{c}4.374 \\
(8.525)\end{array}$ \\
\hline DFG grants & $\begin{array}{c}0.0732 \\
(0.608)\end{array}$ & $\begin{array}{r}0.0775 \\
(0.665)\end{array}$ & $\begin{array}{c}0.0661 \\
(0.503)\end{array}$ & $\begin{array}{c}0.0496 \\
(0.630)\end{array}$ & $\begin{array}{c}0.0311 \\
(0.285)\end{array}$ & $\begin{array}{c}0.120 \\
(0.713)\end{array}$ & $\begin{array}{c}0.153 \\
(0.817)\end{array}$ \\
\hline Months abroad & $\begin{array}{r}9.825 \\
(23.10)\end{array}$ & $\begin{array}{r}8.657 \\
(20.08)\end{array}$ & $\begin{array}{c}11.71 \\
(27.18)\end{array}$ & $\begin{array}{c}7.323 \\
(15.77)\end{array}$ & $\begin{array}{c}11.20 \\
(26.04)\end{array}$ & $\begin{array}{c}10.69 \\
(25.15)\end{array}$ & $\begin{array}{c}12.98 \\
(29.77)\end{array}$ \\
\hline Mobility & $\begin{array}{c}1.454 \\
(1.522)\end{array}$ & $\begin{array}{c}1.415 \\
(1.480)\end{array}$ & $\begin{array}{c}1.518 \\
(1.585)\end{array}$ & $\begin{array}{c}1.145 \\
(1.345)\end{array}$ & $\begin{array}{c}1.405 \\
(1.526)\end{array}$ & $\begin{array}{c}1.828 \\
(1.578)\end{array}$ & $\begin{array}{c}1.798 \\
(1.691)\end{array}$ \\
\hline Interim professor & $\begin{array}{c}0.111 \\
(0.523)\end{array}$ & $\begin{array}{c}0.123 \\
(0.601)\end{array}$ & $\begin{array}{c}0.0914 \\
(0.363)\end{array}$ & $\begin{array}{c}0.111 \\
(0.676)\end{array}$ & $\begin{array}{c}0.0680 \\
(0.307)\end{array}$ & $\begin{array}{c}0.141 \\
(0.462)\end{array}$ & $\begin{array}{c}0.149 \\
(0.468)\end{array}$ \\
\hline Persons & 805 & 469 & 336 & 256 & 217 & 213 & 119 \\
\hline Person-years & 7,667 & 4,734 & 2,933 & 2,861 & 2,088 & 1,873 & 845 \\
\hline
\end{tabular}

Notes: Mean coefficients; SD in parentheses.

the descriptive data into men and women. Columns 4-7 further split up the data into childless men and women, fathers and mothers.

Column 1 of Table 1 shows that we have 7,667 person-year observations from 805 different researchers. During an average year in our dataset, researchers publish 0.161 (co-author-adjusted) SSCI articles per year. Comparing Columns 2 and 3, men publish about 50 per cent more than women each year (0.185-0.120). Columns 4-7 further differentiate between male and female academics with and without children. Fathers publish the most, followed by childless men, mothers, and childless women. There is not only a gender publication gap but also a gender child gap, with male sociologists having about 50 per cent more children than women (0.650 to 0.429$)$.

\section{Results from Random-Effects Models}

Table 2 shows the results of random-effects regressions. The constant in Model 1 shows that men publish 0.197 SSCI articles annually, while women publish 0.062 fewer articles on average. Hence, without further controls, female sociologists publish about 31 per cent fewer articles. Note that this is virtually the same effect as in the descriptive overview of Table 1 , which is reasonable, as the model does not include controls.

Model 2 includes interaction effects for both men and women with their number of children. With each 
Table 2. Random-effects regression on SSCl productivity (dependent variable: annual number of SSCl journal articles)

\begin{tabular}{|c|c|c|c|c|c|c|c|c|}
\hline & $\begin{array}{l}(1) \\
\text { Gender } \\
\text { gap }\end{array}$ & $\begin{array}{l}(2) \\
\text { Children } \\
\text { added }\end{array}$ & $\begin{array}{l}(3) \\
\text { Experience } \\
\text { added }\end{array}$ & $\begin{array}{l}(4) \\
\text { Publications } \\
\text { added }\end{array}$ & $\begin{array}{l}(5) \\
\text { Mobility } \\
\text { added }\end{array}$ & $\begin{array}{l}(6) \\
\text { Without } \\
\text { children } \\
\text { variable }\end{array}$ & $\begin{array}{l}(7) \\
\text { Women } \\
\text { only }\end{array}$ & $\begin{array}{l}(8) \\
\text { Men } \\
\text { only }\end{array}$ \\
\hline Female & $\begin{array}{l}-0.062^{* * *} \\
(-3.31)\end{array}$ & $\begin{array}{l}-0.058^{* *} \\
(-2.96)\end{array}$ & $\begin{array}{l}-0.062 * * \\
(-3.20)\end{array}$ & $\begin{array}{l}-0.038 * * \\
(-3.26)\end{array}$ & $\begin{array}{l}-0.038^{* *} \\
(-3.26)\end{array}$ & $\begin{array}{l}-0.044 * * * \\
(-4.98)\end{array}$ & & \\
\hline $\begin{array}{l}\text { Men } \times \text { number of } \\
\text { children }(\ln ), t-1\end{array}$ & & $\begin{array}{l}0.060 * \\
(2.20)\end{array}$ & $\begin{array}{l}-0.035 \\
(-1.13)\end{array}$ & $\begin{array}{l}-0.052 * \\
(-2.50)\end{array}$ & $\begin{array}{l}-0.052 * \\
(-2.49)\end{array}$ & & & \\
\hline $\begin{array}{l}\text { Women } \times \text { number of } \\
\text { children }(\ln ), t-1\end{array}$ & & $\begin{array}{l}0.044^{+} \\
(1.82)\end{array}$ & $\begin{array}{l}-0.065^{*} \\
(-2.35)\end{array}$ & $\begin{array}{l}-0.056 * * \\
(-2.69)\end{array}$ & $\begin{array}{l}-0.050 * \\
(-2.39)\end{array}$ & & & \\
\hline Number of children $(\ln ), t-1$ & & & & & & & $\begin{array}{l}-0.050 * \\
(-2.24)\end{array}$ & $\begin{array}{l}-0.052 * \\
(-2.41)\end{array}$ \\
\hline Doctorate, $t-1$ & & & $\begin{array}{l}0.138 * * * \\
(7.34)\end{array}$ & $\begin{array}{l}0.091 * * * \\
(5.46)\end{array}$ & $\begin{array}{l}0.086^{* * *} \\
(5.15)\end{array}$ & $\begin{array}{l}0.063 * * * \\
(4.45)\end{array}$ & $\begin{array}{l}0.076^{* *} \\
(3.05)\end{array}$ & $\begin{array}{l}0.094^{* * *} \\
(4.29)\end{array}$ \\
\hline Habilitation, $t-1$ & & & $\begin{array}{l}-0.057^{+} \\
(-1.76)\end{array}$ & $\begin{array}{l}-0.064^{+} \\
(-1.89)\end{array}$ & $\begin{array}{l}-0.048 \\
(-1.40)\end{array}$ & $\begin{array}{l}-0.070 * * \\
(-2.68)\end{array}$ & $\begin{array}{l}-0.060 \\
(-1.61)\end{array}$ & $\begin{array}{l}-0.046 \\
(-0.98)\end{array}$ \\
\hline Years in academia $(\ln ), t-1$ & & & $\begin{array}{l}0.034 * * * \\
(4.40)\end{array}$ & $\begin{array}{l}-0.023 * \\
(-2.25)\end{array}$ & $\begin{array}{l}-0.023^{*} \\
(-2.21)\end{array}$ & $\begin{array}{l}-0.035 * * * \\
(-4.64)\end{array}$ & $\begin{array}{l}-0.028 * \\
(-2.39)\end{array}$ & $\begin{array}{l}-0.017 \\
(-1.10)\end{array}$ \\
\hline SSCI journal articles $(\ln ), t-1$ & & & & $\begin{array}{l}0.212 * * * \\
(12.37)\end{array}$ & $\begin{array}{l}0.212 * * * \\
(12.41)\end{array}$ & $\begin{array}{l}0.227 * * * \\
(11.63)\end{array}$ & $\begin{array}{l}0.173 * * * \\
(5.95)\end{array}$ & $\begin{array}{l}0.227^{* * *} \\
(10.75)\end{array}$ \\
\hline Books $(\ln ), t-1$ & & & & $\begin{array}{l}-0.032^{+} \\
(-1.71)\end{array}$ & $\begin{array}{l}-0.029 \\
(-1.48)\end{array}$ & $\begin{array}{l}-0.034^{+} \\
(-1.83)\end{array}$ & $\begin{array}{l}-0.031 \\
(-1.52)\end{array}$ & $\begin{array}{l}-0.028 \\
(-1.10)\end{array}$ \\
\hline Book chapters $(\ln ), t-1$ & & & & $\begin{array}{l}-0.053 * * * \\
(-4.12)\end{array}$ & $\begin{array}{l}-0.054 * * * \\
(-4.05)\end{array}$ & $\begin{array}{l}-0.035 * * * \\
(-3.30)\end{array}$ & $\begin{array}{l}-0.012 \\
(-1.06)\end{array}$ & $\begin{array}{l}-0.078 * * * \\
(-4.32)\end{array}$ \\
\hline Awards $(\ln ), t-1$ & & & & $\begin{array}{l}0.176^{* * *} \\
(4.22)\end{array}$ & $\begin{array}{l}0.172 * * * \\
(4.02)\end{array}$ & $\begin{array}{l}0.121^{* *} \\
(3.20)\end{array}$ & $\begin{array}{l}0.201 * * \\
(3.13)\end{array}$ & $\begin{array}{l}0.158 * * \\
(3.07)\end{array}$ \\
\hline Co-authors $(\ln ), t-1$ & & & & $\begin{array}{c}0.013^{+} \\
(1.79)\end{array}$ & $\begin{array}{l}0.016^{*} \\
(2.20)\end{array}$ & $\begin{array}{l}0.021 * * * \\
(3.41)\end{array}$ & $\begin{array}{c}0.002 \\
(0.28)\end{array}$ & $\begin{array}{l}0.025^{*} \\
(2.48)\end{array}$ \\
\hline $\begin{array}{l}\text { International } \\
\quad \text { publications }(\ln ), t-1\end{array}$ & & & & $\begin{array}{l}0.054 * * * \\
(4.65)\end{array}$ & $\begin{array}{l}0.042^{* * *} \\
(3.53)\end{array}$ & $\begin{array}{l}0.032 * * \\
(2.98)\end{array}$ & $\begin{array}{l}0.039 * * \\
(2.82)\end{array}$ & $\begin{array}{l}0.042 \% \\
(2.48)\end{array}$ \\
\hline DFG grants $(\ln ), t-1$ & & & & $\begin{array}{c}0.147 \\
(1.25)\end{array}$ & $\begin{array}{c}0.151 \\
(1.28)\end{array}$ & $\begin{array}{c}0.080 \\
(0.93)\end{array}$ & $\begin{array}{r}0.115 \\
(1.12)\end{array}$ & $\begin{array}{r}0.158 \\
(0.97)\end{array}$ \\
\hline Months abroad $(\ln ), t-1$ & & & & & $\begin{array}{l}0.017 * * * \\
(3.47)\end{array}$ & $\begin{array}{l}0.018 * * * \\
(4.08)\end{array}$ & $\begin{array}{l}0.018 * * \\
(2.74)\end{array}$ & $\begin{array}{l}0.020 * * \\
(2.63)\end{array}$ \\
\hline Mobility $(\ln ), t-1$ & & & & & $\begin{array}{l}-0.004 \\
(-0.40)\end{array}$ & $\begin{array}{r}0.001 \\
(0.12)\end{array}$ & $\begin{array}{l}-0.006 \\
(-0.52)\end{array}$ & $\begin{array}{l}-0.006 \\
(-0.35)\end{array}$ \\
\hline Interim professor $(\ln ), t-1$ & & & & & $\begin{array}{l}-0.049^{*} \\
(-2.16)\end{array}$ & $\begin{array}{l}-0.032 \\
(-1.40)\end{array}$ & $\begin{array}{l}-0.059 \\
(-1.57)\end{array}$ & $\begin{array}{l}-0.041 \\
(-1.38)\end{array}$ \\
\hline Constant & $\begin{array}{l}0.197 * * * \\
(14.72)\end{array}$ & $\begin{array}{l}0.200 * * * \\
(14.59)\end{array}$ & $\begin{array}{l}0.213 * * * \\
(14.85)\end{array}$ & $\begin{array}{l}0.194 * * * \\
(22.08)\end{array}$ & $\begin{array}{c}0.193 * * * \\
(22.01)\end{array}$ & $\begin{array}{c}0.196 * * * \\
(25.14)\end{array}$ & $\begin{array}{l}0.151 * * * \\
(15.08)\end{array}$ & $\begin{array}{l}0.193 * * * \\
(22.14)\end{array}$ \\
\hline$R^{2}$ overall & 0.005 & 0.004 & 0.029 & 0.159 & 0.162 & 0.150 & 0.141 & 0.169 \\
\hline$R^{2}$ within & 0.000 & 0.005 & 0.029 & 0.008 & 0.009 & 0.005 & 0.004 & 0.013 \\
\hline$R^{2}$ between & 0.013 & 0.007 & 0.047 & 0.536 & 0.533 & 0.541 & 0.535 & 0.519 \\
\hline$N$ (persons) & 729 & 729 & 729 & 729 & 729 & 1,109 & 297 & 432 \\
\hline$N$ (person-years) & 6,846 & 6,846 & 6,846 & 6,846 & 6,846 & 10,581 & 2,592 & 4,254 \\
\hline
\end{tabular}

Note: $t$ statistics in parentheses.

${ }^{+} P<0.1, * P<0.05, * * P<0.01, * * * P<0.001$.

$\log$ increase in the number of children, men publish 0.06 more annual SSCI articles and women 0.044. As parents tend to be more experienced researchers and have more publications as such, we have to additionally control for their experience, which Model 3 does by controlling for a researcher's career stage and seniority. The effect of children then turns negative for both genders but only significantly so for women and almost twice as much as for men. 
Table 3. Fixed-effects regression on SSCl productivity (dependent variable: annual number of SSCl journal articles)

\begin{tabular}{|c|c|c|c|c|c|c|c|c|}
\hline & $\begin{array}{l}(1) \\
\text { Women } \\
\text { only }\end{array}$ & $\begin{array}{l}(2) \\
\text { Men } \\
\text { only }\end{array}$ & $\begin{array}{l}(3) \\
\text { Women } \\
\text { only }\end{array}$ & $\begin{array}{l}(4) \\
\text { Men } \\
\text { only }\end{array}$ & $\begin{array}{l}(5) \\
\text { Women } \\
\text { only }\end{array}$ & $\begin{array}{l}(6) \\
\text { Men } \\
\text { only }\end{array}$ & $\begin{array}{l}\text { (7) } \\
\text { Women } \\
\text { only }\end{array}$ & $\begin{array}{l}(8) \\
\text { Men } \\
\text { only }\end{array}$ \\
\hline Number of children $(\ln ), t-1$ & $\begin{array}{l}0.067^{*} \\
(2.44)\end{array}$ & $\begin{array}{l}0.119 * * * \\
(3.44)\end{array}$ & $\begin{array}{l}-0.062^{+} \\
(-1.74)\end{array}$ & $\begin{array}{l}-0.031 \\
(-0.68)\end{array}$ & $\begin{array}{l}-0.080 * \\
(-2.10)\end{array}$ & $\begin{array}{l}-0.030 \\
(-0.62)\end{array}$ & $\begin{array}{l}-0.079 * \\
(-2.02)\end{array}$ & $\begin{array}{l}-0.035 \\
(-0.73)\end{array}$ \\
\hline Doctorate, $t-1$ & & & $\begin{array}{l}0.098 * * * \\
(4.04)\end{array}$ & $\begin{array}{l}0.120 * * * \\
(3.91)\end{array}$ & $\begin{array}{l}0.105^{* *} \\
(3.10)\end{array}$ & $\begin{array}{l}0.127^{* * *} \\
(3.84)\end{array}$ & $\begin{array}{l}0.102 * * \\
(3.08)\end{array}$ & $\begin{array}{l}0.112 * * * \\
(3.43)\end{array}$ \\
\hline Habilitation, $t-1$ & & & $\begin{array}{l}-0.059 \\
(-1.55)\end{array}$ & $\begin{array}{l}-0.065 \\
(-1.51)\end{array}$ & $\begin{array}{l}-0.050 \\
(-1.14)\end{array}$ & $\begin{array}{l}-0.037 \\
(-0.76)\end{array}$ & $\begin{array}{l}-0.035 \\
(-0.71)\end{array}$ & $\begin{array}{c}-0.018 \\
(-0.37)\end{array}$ \\
\hline Years in academia $(\ln ), t-1$ & & & $\begin{array}{l}0.043 * * * \\
(3.73)\end{array}$ & $\begin{array}{l}0.058 * * * \\
(4.92)\end{array}$ & $\begin{array}{l}0.039 * * \\
(2.65)\end{array}$ & $\begin{array}{l}0.075 * * * \\
(3.44)\end{array}$ & $\begin{array}{l}0.035 \% \\
(2.41)\end{array}$ & $\begin{array}{l}0.068 * * \\
(3.12)\end{array}$ \\
\hline SSCI journal articles $(\ln ), t-1$ & & & & & $\begin{array}{l}-0.206 * * * \\
(-5.03)\end{array}$ & $\begin{array}{l}-0.138 * * * \\
(-4.22)\end{array}$ & $\begin{array}{l}-0.207 * * * \\
(-5.04)\end{array}$ & $\begin{array}{l}-0.134 * * * \\
(-4.03)\end{array}$ \\
\hline Books $(\ln ), t-1$ & & & & & $\begin{array}{l}0.022 \\
(0.54)\end{array}$ & $\begin{array}{l}-0.016 \\
(-0.42)\end{array}$ & $\begin{array}{c}0.019 \\
(0.46)\end{array}$ & $\begin{array}{l}-0.010 \\
(-0.28)\end{array}$ \\
\hline Book chapters $(\ln ), t-1$ & & & & & $\begin{array}{c}-0.018 \\
(-0.84)\end{array}$ & $\begin{array}{l}-0.061 \% \\
(-2.43)\end{array}$ & $\begin{array}{l}-0.012 \\
(-0.57)\end{array}$ & $\begin{array}{l}-0.067^{*} \\
(-2.58)\end{array}$ \\
\hline Awards $(\ln ), t-1$ & & & & & $\begin{array}{r}0.152 \\
(1.33)\end{array}$ & $\begin{array}{c}0.111 \\
(1.34)\end{array}$ & $\begin{array}{c}0.140 \\
(1.20)\end{array}$ & $\begin{array}{c}0.111 \\
(1.30)\end{array}$ \\
\hline Co-authors (ln), $t-1$ & & & & & $\begin{array}{r}0.029 \\
(1.42)\end{array}$ & $\begin{array}{c}0.022 \\
(1.11)\end{array}$ & $\begin{array}{r}0.030 \\
(1.51)\end{array}$ & $\begin{array}{r}0.022 \\
(1.13)\end{array}$ \\
\hline $\begin{array}{l}\text { International publications } \\
\quad(\ln ), t-1\end{array}$ & & & & & $\begin{array}{l}0.062 * \\
(2.44)\end{array}$ & $\begin{array}{l}0.087^{* * *} \\
(3.57)\end{array}$ & $\begin{array}{c}0.051^{+} \\
(1.96)\end{array}$ & $\begin{array}{l}0.074 * * \\
(3.19)\end{array}$ \\
\hline DFG grants $(\ln ), t-1$ & & & & & $\begin{array}{c}0.076 \\
(1.19)\end{array}$ & $\begin{array}{r}0.222 \\
(1.11)\end{array}$ & $\begin{array}{r}0.094 \\
(1.43)\end{array}$ & $\begin{array}{r}0.222 \\
(1.13)\end{array}$ \\
\hline Months abroad $(\ln ), t-1$ & & & & & & & $\begin{array}{l}0.037^{*} \\
(2.33)\end{array}$ & $\begin{array}{r}0.031 \\
(1.59)\end{array}$ \\
\hline Mobility $(\ln ), t-1$ & & & & & & & $\begin{array}{l}-0.038 \\
(-1.09)\end{array}$ & $\begin{array}{c}0.061^{+} \\
(1.74)\end{array}$ \\
\hline Interim professor $(\ln ), t-1$ & & & & & & & $\begin{array}{l}-0.074 \\
(-1.04)\end{array}$ & $\begin{array}{l}-0.105^{*} \\
(-2.56)\end{array}$ \\
\hline Constant & $\begin{array}{l}0.139 * * * \\
(40.71)\end{array}$ & $\begin{array}{l}0.196 * * * \\
(714.56)\end{array}$ & $\begin{array}{l}0.132 * * * \\
(35.32)\end{array}$ & $\begin{array}{l}0.197 * * * \\
(206.66)\end{array}$ & $\begin{array}{l}0.101 * * * \\
(12.71)\end{array}$ & $\begin{array}{l}0.197 * * * \\
(102.88)\end{array}$ & $\begin{array}{l}0.095 * * * \\
(11.45)\end{array}$ & $\begin{array}{l}0.196 * * * \\
(95.82)\end{array}$ \\
\hline$R^{2}$ overall & 0.001 & 0.000 & 0.027 & 0.020 & 0.000 & 0.018 & 0.006 & 0.020 \\
\hline$R^{2}$ within & 0.002 & 0.006 & 0.028 & 0.031 & 0.053 & 0.049 & 0.058 & 0.053 \\
\hline$R^{2}$ between & 0.000 & 0.001 & 0.045 & 0.017 & 0.028 & 0.000 & 0.001 & 0.002 \\
\hline$N$ (persons) & 297 & 432 & 297 & 432 & 297 & 432 & 297 & 432 \\
\hline$N$ (person-years) & 2,592 & 4,254 & 2,592 & 4,254 & 2,592 & 4,254 & 2,592 & 4,254 \\
\hline
\end{tabular}

Note: $t$ statistics in parentheses.

${ }^{+} P<0.1,{ }^{*} P<0.05, * * P<0.01, * * * P<0.001$.

Even though having children decreases the number of publications for women almost twice as much as for men, controlling for this effect hardly changes the female publication gap, as the coefficient of the female dummy shows. However, the female publication gap could be explained by prior academic performance. Therefore, Model 4 controls for accumulated publications, awards, co-authorships, international publications, and third-party funding. After controlling for this, having children significantly decreases the number of publications for men as well. This means that, on average, both male and female academics experience a decline of publications after becoming parents, relative to what would be expected for their level of experience, prior scientific output, etc. The constant in Model 4 shows that childless men with average research experience publish 0.194 SSCI articles annually, while childless women publish $0.194-0.038=0.156$ articles. Controlling for prior performance, therefore, reduces the female publication gap to about 20 per cent, down from 31 per cent in Model 1. This suggests that differences in prior accumulated achievement, as well as the 
Table 4. Fixed-effects regression SSCl productivity, testing effects of prior academic performance (dependent variable: annual number of $\mathrm{SSCl}$ journal articles)

\begin{tabular}{|c|c|c|c|c|}
\hline & $\begin{array}{l}(1) \\
\text { Women }\end{array}$ & $\begin{array}{l}(2) \\
\text { Men }\end{array}$ & $\begin{array}{l}(3) \\
\text { Women }\end{array}$ & $\begin{array}{l}(4) \\
\text { Men }\end{array}$ \\
\hline Number of children $(\ln ), t-1$ & $\begin{array}{l}-0.147 * * \\
(-2.93)\end{array}$ & $\begin{array}{c}0.041 \\
(0.66)\end{array}$ & $\begin{array}{l}-0.093 * \\
(-2.84)\end{array}$ & $\begin{array}{r}0.029 \\
(0.73)\end{array}$ \\
\hline Doctorate, $t-1$ & $\begin{array}{l}0.110 * * \\
(3.20)\end{array}$ & $\begin{array}{l}0.096^{* *} \\
(2.99)\end{array}$ & $\begin{array}{l}0.068 * * \\
(3.19)\end{array}$ & $\begin{array}{l}0.060 * * \\
(3.23)\end{array}$ \\
\hline Habilitation, $t-1$ & $\begin{array}{l}-0.036 \\
(-0.73)\end{array}$ & $\begin{array}{l}-0.037 \\
(-0.93)\end{array}$ & $\begin{array}{l}-0.018 \\
(-0.55)\end{array}$ & $\begin{array}{c}-0.026 \\
(-1.09)\end{array}$ \\
\hline Years in academia $(\ln ), t-1$ & $\begin{array}{l}0.037^{*} \\
(2.40)\end{array}$ & $\begin{array}{l}0.052 * * \\
(2.92)\end{array}$ & $\begin{array}{l}0.026 * * \\
(2.67)\end{array}$ & $\begin{array}{l}0.033 * * \\
(3.03)\end{array}$ \\
\hline SSCI journal articles $(\ln ), t-1$ & $\begin{array}{l}-0.207^{* * *} \\
(-3.93)\end{array}$ & $\begin{array}{l}-0.139 * * \\
(-2.99)\end{array}$ & $\begin{array}{l}-0.131^{* * *} \\
(-4.08)\end{array}$ & $\begin{array}{l}-0.105 * * * \\
(-3.94)\end{array}$ \\
\hline Books $(\ln ), t-1$ & $\begin{array}{c}0.021 \\
(0.41)\end{array}$ & $\begin{array}{c}0.013 \\
(0.36)\end{array}$ & $\begin{array}{r}0.018 \\
(0.58)\end{array}$ & $\begin{array}{c}0.011 \\
(0.47)\end{array}$ \\
\hline Book chapters $(\ln ), t-1$ & $\begin{array}{l}-0.009 \\
(-0.36)\end{array}$ & $\begin{array}{l}-0.051^{*} \\
(-2.09)\end{array}$ & $\begin{array}{c}-0.008 \\
(-0.48)\end{array}$ & $\begin{array}{l}-0.031 \% \\
(-2.19)\end{array}$ \\
\hline Awards $(\ln ), t-1$ & $\begin{array}{l}-0.017 \\
(-0.09)\end{array}$ & $\begin{array}{c}0.132 \\
(1.26)\end{array}$ & $\begin{array}{r}0.007 \\
(0.08)\end{array}$ & $\begin{array}{c}0.079 \\
(1.25)\end{array}$ \\
\hline Co-authors $(\ln ), t-1$ & $\begin{array}{c}0.037^{+} \\
(1.82)\end{array}$ & $\begin{array}{c}0.019 \\
(0.99)\end{array}$ & $\begin{array}{c}0.022^{+} \\
(1.72)\end{array}$ & $\begin{array}{r}0.017 \\
(1.47)\end{array}$ \\
\hline International publications $(\ln ), t-1$ & $\begin{array}{c}0.039 \\
(1.24)\end{array}$ & $\begin{array}{l}0.081 * * \\
(3.20)\end{array}$ & $\begin{array}{r}0.026 \\
(1.28)\end{array}$ & $\begin{array}{l}0.051 * * * \\
(3.36)\end{array}$ \\
\hline DFG grants $(\ln ), t-1$ & $\begin{array}{c}0.034 \\
(0.22)\end{array}$ & $\begin{array}{l}0.626^{*} \\
(2.57)\end{array}$ & $\begin{array}{c}-0.001 \\
(-0.01)\end{array}$ & $\begin{array}{l}0.267 * * \\
(2.77)\end{array}$ \\
\hline Months abroad $(\ln ), t-1$ & $\begin{array}{l}0.036^{*} \\
(2.28)\end{array}$ & $\begin{array}{c}0.023 \\
(1.35)\end{array}$ & $\begin{array}{l}0.024^{*} \\
(2.40)\end{array}$ & $\begin{array}{c}0.011 \\
(1.09)\end{array}$ \\
\hline Mobility (ln), $t-1$ & $\begin{array}{c}-0.048 \\
(-1.18)\end{array}$ & $\begin{array}{c}0.062^{+} \\
(1.75)\end{array}$ & $\begin{array}{l}-0.030 \\
(-1.22)\end{array}$ & $\begin{array}{c}0.036^{+} \\
(1.69)\end{array}$ \\
\hline Interim professor $(\ln ), t-1$ & $\begin{array}{c}-0.073 \\
(-0.97)\end{array}$ & $\begin{array}{l}-0.099^{*} \\
(-2.39)\end{array}$ & $\begin{array}{l}-0.050 \\
(-1.07)\end{array}$ & $\begin{array}{l}-0.057^{*} \\
(-2.13)\end{array}$ \\
\hline Number of children $(\ln ), t-1 \times \mathrm{SSCI}$ journal articles $(\ln ), t-1$ & $\begin{array}{l}-0.015 \\
(-0.21)\end{array}$ & $\begin{array}{c}-0.012 \\
(-0.19)\end{array}$ & $\begin{array}{c}-0.016 \\
(-0.36)\end{array}$ & $\begin{array}{c}0.004 \\
(0.11)\end{array}$ \\
\hline Number of children $(\ln ), t-1 \times$ books $(\ln ), t-1$ & $\begin{array}{c}-0.013 \\
(-0.26)\end{array}$ & $\begin{array}{c}-0.014 \\
(-0.23)\end{array}$ & $\begin{array}{c}-0.005 \\
(-0.17)\end{array}$ & $\begin{array}{c}-0.011 \\
(-0.30)\end{array}$ \\
\hline Number of children $(\ln ), t-1 \times$ book chapters $(\ln ), t-1$ & $\begin{array}{l}-0.015 \\
(-0.46)\end{array}$ & $\begin{array}{c}0.001 \\
(0.02)\end{array}$ & $\begin{array}{c}-0.011 \\
(-0.54)\end{array}$ & $\begin{array}{c}-0.002 \\
(-0.09)\end{array}$ \\
\hline Number of children $(\ln ), t-1 \times$ awards $(\ln ), t-1$ & $\begin{array}{c}0.512 \\
(1.54)\end{array}$ & $\begin{array}{l}-0.000 \\
(-0.00)\end{array}$ & $\begin{array}{c}0.285^{+} \\
(1.86)\end{array}$ & $\begin{array}{c}-0.007 \\
(-0.10)\end{array}$ \\
\hline Number of children $(\ln ), t-1 \times$ international publications $(\ln ), t-1$ & $\begin{array}{c}0.033 \\
(0.68)\end{array}$ & $\begin{array}{l}-0.008 \\
(-0.22)\end{array}$ & $\begin{array}{c}0.021 \\
(0.66)\end{array}$ & $\begin{array}{l}-0.003 \\
(-0.12)\end{array}$ \\
\hline Number of children $(\ln ), t-1 \times$ DFG grants $(\ln ), t-1$ & $\begin{array}{c}0.088 \\
(0.57)\end{array}$ & $\begin{array}{l}-0.570 * * \\
(-2.69)\end{array}$ & $\begin{array}{c}0.083 \\
(1.10)\end{array}$ & $\begin{array}{l}-0.251^{* *} \\
(-2.85)\end{array}$ \\
\hline Number of children $(\ln ), t-1 \times$ mobility $(\ln ), t-1$ & $\begin{array}{c}0.034 \\
(0.65)\end{array}$ & $\begin{array}{l}-0.016 \\
(-0.29)\end{array}$ & $\begin{array}{c}0.024 \\
(0.72)\end{array}$ & $\begin{array}{c}-0.012 \\
(-0.36)\end{array}$ \\
\hline Constant & $\begin{array}{l}0.228 * * * \\
(4.95)\end{array}$ & $\begin{array}{l}0.196^{* * * *} \\
(4.92)\end{array}$ & $\begin{array}{l}0.147^{* * * *} \\
(5.35)\end{array}$ & $\begin{array}{l}0.135^{* * *} \\
(5.47)\end{array}$ \\
\hline$R^{2}$ overall & 0.005 & 0.030 & 0.005 & 0.010 \\
\hline$R^{2}$ within & 0.065 & 0.068 & 0.069 & 0.063 \\
\hline$R^{2}$ between & 0.005 & 0.002 & 0.003 & 0.007 \\
\hline$N$ (persons) & 297 & 432 & 297 & 432 \\
\hline$N$ (person-years) & 2,592 & 4,254 & 2,592 & 4,254 \\
\hline
\end{tabular}

Note: $t$ statistics in parentheses.

${ }^{+} P<0.1,{ }^{*} P<0.05, * * P<0.01, * * * P<0.001$. 
negative effect of children, explain part of the female publication gap.

However, Model 5 adds mobility variables, which does not change the influence of children on publication output. We also tested separate interaction effects between mobility and the number of children, with no substantial results (not shown). This indicates that researchers with children do not publish less because they are less mobile.

Model 6 is the same as Model 5, but does not control for the gendered effect of children on publications. Keep in mind that Model 5, after including all controls, shows that childless women publish about 20 per cent less than men $(0.038 / 0.193=19.7 \%)$. Model 6 , which does not include the gendered effect of children on publications, shows that women publish $0.044 / 0.196=22.4$ per cent less than men at the same career stage and similar prior productivity levels. This means that the female publication gap only narrows slightly after controlling for the number of children. It is only 12.3 per cent $(19.7 / 22.4=0.877)$ lower after accounting for the gendered effect of children on publications. Thus, it seems that the effect of childbearing, while significantly decreasing publication output, does not strongly affect the overall female publication gap, which thus exists independently of the effect of children.

Finally, Models 7 and 8 split the regressions by gender. Both models suggest that having children decreases the number of publications similarly for both genders, confirming the results of Model 5. Overall, the results indicate that the effect of children on publication output is similarly negative for men and women. However, while random-effect regressions are focused on group differences as well as individual differences, only fixedeffects regressions show whether the same researcher is more or less productive after having children. This is what we turn to next.

\section{Results from Fixed-Effects Models}

Fixed-effects models cannot identify the effect of timeconstant variables, such as gender. We calculate separate regressions for women (Models 1, 3, 5, and 7) and men (Models 2, 4, 6, and 8). Models 1 and 2 in Table 3 estimate the effect of children without controls, then Models 3 to 8 gradually add controls for seniority, academic achievements, such as prior publications, and mobility.

In the uncontrolled Models 1 and 2 of Table 3, the within-effect of parenthood on research output is positive for both genders. As in the random-effects regressions, this may be because childbirth takes place during the more advanced career stages of untenured researchers when research productivity is generally higher. To correct for this confounding effect, Models 3 and 4 control for seniority. This turns both effects negative but not significantly so (only at $P$ less than 0.1 for women). However, controlling for measures of prior performance in Models 5-8 turns the effect of childbirth on productivity significantly negative for women and insignificantly negative for men. Substantively, this means that having children depresses a woman's subsequent research more than twice as much as a man's (compare the effect sizes of Models 5 and 6 as well as Models 7 and 8).

That the effect of childbearing on publications changes after controlling for prior academic achievements, suggests that the productivity of women with lower achievements declines disproportionately after childbirth. Table 4 tests the interaction between the effect of having children and seven measures of prior academic achievement, specifically the accumulated number of SSCI journal articles (1), published books (2), book chapters (3), academic awards (4), international publications (5), DFG grants (6) and mobility (7). Since these predictor variables are not mean-centered, the main effects of the number of children in Table 4 show, due to the inclusion of interaction terms, the effect of childbirth on productivity for women (Model 1) and men (Model 2) with very low productivity (zero on all measures). Models 3 (women) and 4 (men) are the same as Models 1 and 2 but use a logged dependent variable for comparison.

The main effect in Model 1 shows that when a woman's pre-birth measures of productivity are zero, then each $\log$ increase in the number of children further decreases her SSCI publications by 0.147 annually. To put this into perspective, the main effect of Table 3 showed that a woman with average measures of academic achievement (mean values on each performance variable) can expect a decrease of 0.079 annual SSCI publications with each $\log$ increase of children. This means that parenthood decreases publications twice as much for a woman with low academic achievements compared with a woman with average performance. By calculating margins, we can compute that a woman who has one (instead of zero) SSCI article, book, book chapter, award, international publication, DFG grant, and change of place at the same career stage, publishes 0.038 fewer articles annually after having a child, compared with the reduction of 0.147 articles for a woman with lowest prior productivity. The number of publications by a mother with lower measures of academic achievement in this sense declines almost four times as much as that of a mother with higher academic achievements. 
For a typical man, the effect of parenthood hardly depends on prior academic success. This is in line with theoretical expectations based on male benefits from traditional gender roles within the household.

None of the interaction effects are significant, except academic awards for females in Model 3 (at $P$ less than 0.10). This means that the detrimental effect of having children on productivity is slightly reduced when women have accumulated more academic awards, which suggests that the signalling function of academic awards may encourage female researchers to keep publishing after childbirth.

To facilitate the interpretation of this interaction effect, Figure 1 shows how having children relates to the number of SSCI publications (y-axis), conditional on the number of awards women have received so far ( $\mathrm{x}$-axis, based on Model 3 in Table 4). The figure also displays the lower and upper limits of the effect's 95 per cent confidence interval. Effects are significant at the 5-per cent level when the interval does not cover the line where y is zero. The dashed vertical line plots a women's mean logged number of awards. As can be seen, having children is negatively associated with publication output if women received an average or below-average number of awards. If, in contrast, women have obtained more than an above-average number of awards, then the effect of children on publication output becomes insignificant.
This suggests that women keep up their publications after childbirth when award-giving committees have encouraged them to believe in their potential for a scientific career.

\section{Robustness Checks}

Tables A1-A3 present alternative specifications as robustness checks. Table A1 replicates the main results (Models 7 and 8 in Table 3) from the fixed-effects analysis using a 2-year lag of the number of children (see Models 1 and 2 in Table A1). As can be seen, using a 2-year lag creates even stronger negative effects of children on the publications of women (Model 1), while the effect for men remains insignificant. This suggests that an increase in the number of children reduces publications for at least 2 years. We also tested a 3-year lag (not shown, available upon request). Its effects remained negative but were insignificant. The effect of children on research productivity, therefore, operates most strongly with a 2-year lag, conceivably because differences in productivity need time to affect measurable output.

The subsequent models use a logged dependent variable with a 1-year lag (Models 3 and 4) and a 2-year lag (Models 7 and 8). For women, an increase in the number of children by one child corresponds to a decrease in

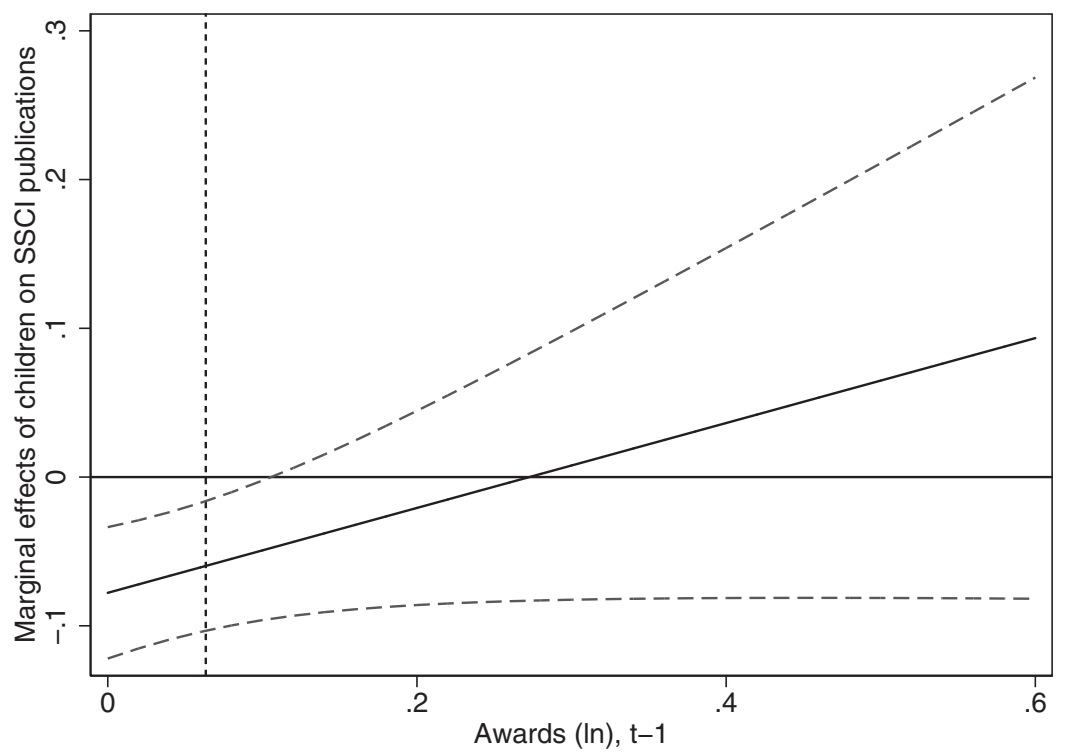

Figure 1. Marginal effects of the number of children on SSCl publications ( $y$-axis), conditional on the number of awards ( $x$-axis) for women

Notes: Based on Model 3 in Table 4. All covariates fixed at their means. Dashed vertical line: women's mean number of awards. Dashed curves: lower and upper limits of the effect's $95 \%$ confidence interval. 
annual SSCI publications by about 5.2 per cent in the following year (Model 3), or about 6.7 per cent 2 years later (Model 5).

Table A2 replicates the main results (Models 7 and 8 of Table 3) using fixed-effects individual-specific slopes (FEIS) regressions. Ludwig and Brüderl (2018) have argued that conventional fixed-effects models neglect differences in the growth of career performance, which in our case are differences in the growth of academic performance within careers. Since this might be an important selection criterion, we estimate FEIS models to test the robustness of the main regressions. FEIS models need to specify a slope function that models the selection process and interacts with time-constant individual heterogeneity. We base its function on two of the most significant measures of prior performance: total number of SSCI publications and number of awards. The models in Table A2 estimate the 1-year and 2-year lags of the children variable both for the non-logged (Models 1-4) and the logged dependent variable (Models 5-8). As can be seen, the FEIS models replicate the children's effect with regard to both effect sizes and statistical significance.

Table A3 replicates Table 3 using the children variable coded as dummies (lagged by 2 years). The dummy coding shows whether the effect is linear (see Model 7): women who give birth to their first child publish 0.068 fewer SSCI articles 2 years later compared with childless women, all else being equal. Women who give birth to a second child face a decline in output by about 0.126 compared with childless women. That two children depress publications almost twice as much as one child indicates the absence of 'economies of scale'. Thus, increasing the number of children has an almost linearly negative effect on the productivity of women. Having three or more children, finally, points to a decline of publications as well but is insignificant, probably due to the small number of women in the sample with three or more children. For male productivity, according to Model 8 , children generally make no difference to productivity. This gender difference is significant at $P<.05$ for the first two children. ${ }^{3}$

\section{Conclusions}

This study has analyzed how parenthood affects the academic publications of men and women. We obtain four main results, which contribute to the existing literature in four ways. Our first main result is that the estimates from random- and fixed-effects regressions point in different directions. Random-effects models (which mix between- and within-effects) indicate that children depress the publications of men and women similarly. Using fixed-effects regressions, however, we find that children depress the publications of women but not of men. This result advances prior research, which mainly drew on between-level effects and thus focused on differences between groups of parents and non-parents, while unable to show how children affect the publications of the same researcher. The random-effects models indicate that men and women with children each have fewer publications than otherwise similar childless academics. However, the within-effect indicates that a statistically typical father maintains his level of productivity, while a statistically average mother does not. Mothers who have been granted academic awards are also able to maintain their prior level of publications; in this sense, an intrafemale 'Mathew' effect exists, since women who received more awards experience a smaller decline in publications after having children (Merton, 1968).

Second, while we find that, after controlling for career stage and prior research achievement, children have a negative effect on productivity, parents indeed have more publications than childless men and women on a purely descriptive level. This means that mothers and fathers publish more than childless women and men but less than childless women and men at similar career stages and less than what is to be expected, given their pre-childbirth achievements and career stage. These results contradict the prominent finding in the literature, that there is no association between publication output and childrearing responsibilities for women in academia (also cf. Cole and Zuckerman, 1987: p. 125; Hamovitch and Morgenstern, 1977: p. 643; Sax et al., 2002: p. 43). Indeed, we find that exactly such a relationship and suggest that prior studies overlooked it because they have not adequately controlled for career stage, prior achievements, and between-effects versus within-effects (cf. the models in Sax et al., 2002: p. 433f.; Stack, 2004: p. 911f.; Joecks, Pull and Backes-Gellner, 2014: p. 528).

Third, many studies that do not find a relationship between childrearing and publication productivity, argue that this is because highly motivated women eliminate everything except work and children from their lives, so that they keep on publishing after having children (Cole and Zuckerman, 1987: p. 125; Fox, 2005: p. 145; Joecks, Pull and Backes-Gellner, 2014: p. 520). This explanation fits our results, which show that women who have received more academic acclaim do not experience a decline in research output after having children.

Fourth, while our results show that parenthood depresses the research output of women, they also show that the gendered effect of children on publications explains only some of the gender publication gap. After controlling for seniority and prior performance, we find that women publish 22.4 per cent fewer annual SSCI 
journal articles than men do (Model 6 of Table 2). When additionally controlling for the differential effect of children on the publication output of men and women, we show that this difference drops by about 12 per cent to a publications gap of 19.7 per cent (Model 5 of Table 2). Remarkably, this magnitude of the gender gap is similar to what studies found 40 years ago (Hamovitch and Morgenstern, 1977: p. 643). Note also that our variables explain up to 54 per cent of the between-level productivity of one researcher compared with another (Model 6 in Table 2) but at most 6.8 per cent of the (within-level) intra-individual variation in publications (Table 4, Models 1 and 2). This means that a large share of the variation in publishing activity is due to variation between researchers, while a large share of variation in intra-personal productivity remains unexplained. This suggests that the publication of SSCI articles depends on unobservable and unpredictable circumstances, such as experimental outcomes, research findings, alternative book projects, institutional characteristics, personal networks, and unpredictable review processes. The number of children, in fact, is therefore only a small part of the puzzle of how much researchers publish.

Our results are in line with studies showing that the effect of children on women cannot fully account for observed gender gaps in research productivity (Hargens, McCann and Reskin, 1978: p. 159; Stack, 2004: p. 912). This concurs with studies that argue that no significant gender gap exists after gender-specific effects of children on publication output are taken into account (Hunter and Leahey, 2010: p. 447). Overall, we observe that women publish less than men each year, even if we compare men and women at the same career stage and with similar prior academic achievements. We initially supposed that this is because children lower the productivity of women more than of men. However, this only explains a small part of the female publication gap, meaning that the lower number of publications by women cannot be explained entirely by them being trapped in earlier career stages or because female productivity suffers much more from childrearing than male productivity does. Therefore, it, remains somewhat of a puzzle why women publish less than men. While we do observe that women have less access to higher career levels and are more burdened through childcare, neither of these two effects fully explains why they publish less than men do.

While our findings answer some important questions of the research literature, they also suffer from some shortcomings, which mainly consist of unobservable heterogeneity. First, our research design cannot explicitly test the causal mechanisms that may explain why mothers experience stronger declines in publications than fathers. Gender may be a proxy for hours spent on childcare, which could be a more direct measure to explain the female publication gap. One mechanism why women publish less after childbirth is that they may spend more time with their children, while men may use parental leave to work on publications (Antecol, Bedard and Stearns, 2018). Another mechanism could be higher earnings of male parters, which provide an incentive to cut back female employment after having children, while men have an incentive to increase their workload while transferring childrearing tasks to their female partner (Becker, 1991 [1981]). Future research has to show whether such a maximization of household income is a better candidate to explain our results than gender roles.

The results of Antecol, Bedard and Stearns (2018) as well as Krapf, Ursprung and Zimmermann (2017) suggest a positive effect of children on the publication output of fathers. Our results do not show a positive but a non-significant negative effect. The non-significance suggests that children do not strongly suppress the productivity of men. Why do our results differ from this research? Krapf et al. as well as Antecol et al. use a quasiexperimental design, which should allow for robust causal inference. However, their findings are based on US economists, in the case of Antecol, Bedard and Stearns (2018) drawn from the top-50 US economic departments. Their results may differ due to different publication cultures within the field of sociology and economics, or because less institutional pressures or competitiveness exists in German sociology as compared with (top-50) US economics departments. Notably, based on our results, one should observe that the more competitive an environment is and the more selected researchers are, the less their productivity should decrease after they have children. This, however, has to be tested through future research.

Second, we can show that parenthood lowers publication output especially for women with fewer academic awards. However, researchers with fewer publications probably leave academia more often, making it more unlikely to sample them. This changes nothing about our central claim, which is about the population of employed researchers in German academic sociology in the year 2013. However, childbirth might decrease publication output to an even stronger degree if we had included researchers who already left this population. In this sense, it is possible that our analysis underestimates how much children depress female productivity so that the effect we report is rather conservative. Another distinction we could not draw is between the age of children. Conceivably, especially young children burden parents, especially mothers. 
Another problem we face is that there is a gendered research specialization within the field of sociology (Leahey, 2006). Probably more men than women specialize in quantitative sociology, which is more prone to SSCI journal article publication. This brings in possible bias that we can only indirectly control by taking the type of publication into account (SSCI, book chapter, and so on). However, future research should also analyze the content of these publications, and focus on areas of specialization as a possible explanatory factor.

These problems underscore the need for future research. We showed that parenthood lowers the publication output of a typical woman but not of a typical man. It may be that women have less time after childbirth or experience a stronger role conflict. It is also possible that women are integrated in research networks of lesser status (Ibarra, 1992) and that this 'closure penalty' (Lutter, 2015 ) in their networks intensifies with children. Future research should, therefore, compare professional networks of male and female academics to explain the gendered effect of children on academic publications.

\section{Notes}

1 This response rate is relatively high. One reason may be that the survey was just a quick email. It did not contain a link to an external online survey; respondents delivered their answer simply by replying with the number and birth years of their children. We tested whether the group of non-respondents have more annual publications than the group of respondents. We neither find this to be the case among men, nor among women, before and after including controls. We therefore conclude that non-response does not systematically bias our outcome variable, namely publication productivity.

2 We collected this data from a database of the DFG (gepris.dfg.de) and merged it into our database through the names of the researchers and their years of funding.

3 This result appears if we estimate the interaction effects of the female dummy with each of the three dummies on the number of children for both genders (not shown but available upon request).

\section{Acknowledgements}

For helpful suggestions and comments on a previous version, we thank Sabine Köszegi and Ingo Rohlfing. The paper benefitted from constructive comments at the MZES colloquium in Mannheim, and from the sociological colloquium at the University of Konstanz. We thank all participants for constructive comments. We also would like to thank all anonymous reviewers for their helpful comments.

\section{References}

Antecol, H., Bedard, K. and Stearns, J. (2018). Equal but inequitable: who benefits from gender-neutral tenure clock stopping policies? American Economic Review, 108, 2420-2441.

Becker, G. (1991 [1981]). A Treatise on the Family. Boston, MA: Harvard University Press.

Blossfeld, H.-P., Drobnic, S. and Rohwer, G. (2001). Spouses' employment careers in (West) Germany. In Blossfeld, H.-P. and Drobnic, S. (Eds.), Careers of Couples in Contemporary Societies. From Male Breadwinner to Dual Earner Families. Oxford: Oxford University Press, pp. 53-76.

Bohannon, J. (2013). Who's afraid of peer review? Science, 342, 60-65.

Boschini, A. and Sjögren, A. (2007). Is team formation gender neutral? Evidence from coauthorship patterns. Journal of Labor Economics, 25, 325-365.

Cole, J. R. and Singer, B. (1991). A theory of limited differences: explaining the productivity puzzle in science. In Zuckerman, H., Cole, J. R. and Bruer, J. T. (Eds.), The Outer Circle: Women in the Scientific Community. New York: Norton, pp. 277-310.

Cole, J. R. and Zuckerman, H. (1987). Marriage, motherhood and research performance in science. Scientific American, 256, 119-125.

Correll, S. J., Benard, S. and Paik, I. (2007). Getting a job: is there a motherhood penalty? American Journal of Sociology, 112, 1297-1339.

Destatis (2018). Bildung und Kultur. Personal an Hochschulen. Fachserie 11 Reihe 4.4. <https://www.destatis.de/DE/ Themen/Gesellschaft-Umwelt/Bildung-Forschung-Kultur/Hoc hschulen/Publikationen/Downloads-Hochschulen/personalhochschulen-2110440177004.pdf?_blob=publicationFile $>$ [accessed 15 November 2019].

Fox, M. F. (2005). Gender, family characteristics, and publication productivity among scientists. Social Studies of Science, 35, 131-150.

Gangl, M. and Ziefle, A. (2015). The making of a good woman: extended parental leave entitlements and mothers' work commitment in Germany. American Journal of Sociology, 121, 511-563.

Giesselmann, M., Hagen, M. and Schunck, R. (2018). Motherhood and mental well-being in Germany: linking a longitudinal life course design and the gender perspective on motherhood. Advances in Life Course Research, 37, 31-41.

Haas, M. and Koeszegi, S. T. (2017). Spiel Mit Mir. Die Konstruktion Von Geschlecht Und Professionalität in Organisationen-Eine Rahmenanalyse. Forum Qualitative Sozialforschung, 18, Art. 5. < http://dx.doi.org/10.17169/fqs18.3.2587>.

Haas, M., Koeszegi, S. T. and Zedlacher, E. (2016). Breaking patterns? How female scientists negotiate their token role in their life stories. Gender, Work \& Organization, 23, 397-413.

Hamovitch, W. and Morgenstern, R. D. (1977). Children and the productivity of academic women. The Journal of Higher Education, 48, 633-645.

Hargens, L. L., McCann, J. C. and Reskin, B. F. (1978). Productivity and reproductivity: fertility and professional achievement among research scientists. Social Forces, 57, 154-163. 
Hunter, L. A. and Leahey, E. (2010). Parenting and research productivity: new evidence and methods. Social Studies of Science, 40, 433-451.

Ibarra, H. (1992). Homophily and differential returns: sex differences in network structure and access in an advertising firm. Administrative Science Quarterly, 37, 422-447.

Jaksztat, S. (2017). Geschlecht Und Wissenschaftliche Produktivität. Erklären Elternschaft Und Wissenschaftliches Sozialkapital Produktivitätsunterschiede Während Der Promotionsphase? Zeitschrift für Soziologie, 46, 347-361.

Joecks, J., Pull, K. and Backes-Gellner, U. (2014). Childbearing and (female) research productivity: a personnel economics perspective on the leaky pipeline. Journal of Business Economics, 84, 517-530.

Jungbauer-Gans, M. and Gross, C. (2013). Determinants of success in university careers: findings from the german academic labor market. Zeitschrift für Soziologie, 42, 74-92.

Krapf, M., Ursprung, H. W. and Zimmermann, C. (2017). Parenthood and productivity of highly skilled labor: evidence from the groves of academe. Journal of Economic Behavior \& Organization, 140, 147-175.

Kyvik, S. (1990). Motherhood and scientific productivity. Social Studies of Science, 20, 149-160.

Kyvik, S. and Teigen, M. (1996). Child care, research collaboration, and gender differences in scientific productivity. Science, Technology \& Human Values, 21, 54-71.

Leahey, E. (2006). Gender differences in productivity: research specialization as a missing link. Gender \& Society, 20, 754-780.

Long, J. S. (1990). The origins of sex differences in science. Social Forces, 68, 1297-1316.

Lotka, A. J. (1926). The frequency distribution of scientific productivity. Journal of the Washington Academy of Sciences, 16, 317-324.

Ludwig, V. and Brüderl, J. (2018). Is there a male marital wage premium? New evidence from the United States. American Sociological Review, 83, 744-770.

Lutter, M. (2015). Do women suffer from network closure? The moderating effect of social capital on gender inequality in a project-based labor market, 1929 to 2010. American Sociological Review, 80, 329-358.

Lutter, M. and Schröder, M. (2016). Who becomes a tenured professor, and why? Panel data evidence from german sociology, 1980-2013. Research Policy, 45, 999-1013.

Mason, M. A., Wolfinger, N. H. and Goulden, M. (2013). Do Babies Matter? Gender and Family in the Ivory Tower. New Brunswick, NJ: Rutgers University Press.

Merton, R. (1968). The Matthew effect in science. Science, 159, 56-63.

Nakhaie, M. R. (2002). Gender differences in publication among university professors in Canada. The Canadian Review of Sociology, 39, 151.

Petit, P. (2007). The effects of age and family constraints on gender hiring discrimination: a field experiment in the French financial sector. Labour Economics, 14, 371-391.

Rivera, L. A. (2017). When two bodies are (not) a problem: gender and relationship status discrimination in academic hiring. American Sociological Review, 82, 1111-1138.

Sax, L. J. et al. (2002). Faculty research productivity: exploring the role of gender and family-related factors. Research in Higher Education, 43, 423-446.

Schulz, F. and Blossfeld, H.-P. (2006). Wie Verändert Sich Die Häusliche Arbeitsteilung Im Eheverlauf? Kölner Zeitschrift für Soziologie und Sozialpsychologie, 58, 23-49.

Sieverding, M. et al. (2018). Can lifestyle preferences help explain the persistent gender gap in academia? The "mothers work less" hypothesis supported for German but not for U.S. early career researchers. PLOS ONE, 13: e0202728. < https:// doi.org/10.1371/journal.pone.0202728>.

Sigle-Rushton, W. and Waldfogel, J. (2007). Motherhood and women's earnings in Anglo-American, Continental European, and Nordic Countries. Feminist Economics, 13, 55-91.

Stack, S. (1994). The effects of gender on publishing: the case of sociology. Sociological Focus, 27, 81-83.

Stack, S. (2002). Gender and scholarly productivity: 1970-2000. Sociological Focus, 35, 285-296.

Stack, S. (2004). Gender, children and research productivity. Research in Higher Education, 45, 891-920.

Toutkoushian, R. K. and Bellas, M. L. (1999). Faculty time allocations and research productivity: gender, race and family effects. The Review of Higher Education, 22, 367-390.

van den Brink, M. and Benschop, Y. (2011). Gender practices in the construction of academic excellence: sheep with five legs. Organization, 19, 507-524.

Wennerås, C. and Wold, A. (1997). Nepotism and sexism in peer-review. Nature, 387, 341.

Xie, Y. and Shauman, K. A. (1998). Sex differences in research productivity: new evidence about an old puzzle. American Sociological Review, 63, 847-870.

Xie, Y. and Shauman, K. A. (2003). Women in Science: Career Processes and Outcomes. Cambridge, MA: Harvard University Press.

Mark Lutter is Professor of Sociology at Wuppertal University. His research interests are economic sociology, social inequality research, social networks. His work appears in journals such as American Sociological Review, European Sociological Review, European Political Science Review, Sociology, Research Policy, or International Sociology.

Martin Schröder is Professor of Sociology at Marburg University. His research interests are political economy, economic sociology, welfare states, varieties of capitalism, morality, well-being and gender inequalities. His work appears in journals such as Social Policy, Economy and Society, European Sociological Review, Social Forces, Research Policy and International Sociology. 


\section{Appendix}

Table A1. Replication of Table 3, Models 7 and 8, using number of children lagged by two years (Models $1+2,5+6)$ and using logged dependent variable (Models 3-6, log of annual number of SSCl journal articles)

\begin{tabular}{|c|c|c|c|c|c|c|}
\hline & $\begin{array}{l}(1) \\
\text { Women } \\
\text { only }\end{array}$ & $\begin{array}{l}(2) \\
\text { Men } \\
\text { only }\end{array}$ & $\begin{array}{l}(3) \\
\text { Women } \\
\text { only (logged } \\
\text { DV) }\end{array}$ & $\begin{array}{l}(4) \\
\text { Men } \\
\text { only (logged } \\
\text { DV) }\end{array}$ & $\begin{array}{l}\text { (5) } \\
\text { Women } \\
\text { only (logged } \\
\text { DV) }\end{array}$ & $\begin{array}{l}(6) \\
\text { Men } \\
\text { only (logged } \\
\text { DV) }\end{array}$ \\
\hline Number of children $(\ln ), t-1$ & & & $\begin{array}{l}-0.052 * \\
(-2.16)\end{array}$ & $\begin{array}{l}-0.011 \\
(-0.39)\end{array}$ & & \\
\hline Number of children $(\ln ), t-2$ & $\begin{array}{l}-0.100 * \\
(-2.44)\end{array}$ & $\begin{array}{c}0.013 \\
(0.25)\end{array}$ & & & $\begin{array}{l}-0.067^{* *} \\
(-2.60)\end{array}$ & $\begin{array}{r}0.018 \\
(0.62)\end{array}$ \\
\hline Doctorate, $t-1$ & $\begin{array}{l}0.088 * \\
(2.52)\end{array}$ & $\begin{array}{l}0.099 * * \\
(2.81)\end{array}$ & $\begin{array}{l}0.063 * * \\
(3.00)\end{array}$ & $\begin{array}{l}0.068 * * * \\
(3.64)\end{array}$ & $\begin{array}{l}0.054 * \\
(2.41)\end{array}$ & $\begin{array}{l}0.059 * * \\
(2.96)\end{array}$ \\
\hline Habilitation, $t-1$ & $\begin{array}{l}-0.049 \\
(-0.94)\end{array}$ & $\begin{array}{c}-0.011 \\
(-0.23)\end{array}$ & $\begin{array}{l}-0.017 \\
(-0.52)\end{array}$ & $\begin{array}{l}-0.018 \\
(-0.69)\end{array}$ & $\begin{array}{l}-0.026 \\
(-0.75)\end{array}$ & $\begin{array}{l}-0.013 \\
(-0.51)\end{array}$ \\
\hline Years in academia $(\ln ), t-1$ & $\begin{array}{l}0.057^{*} \\
(2.28)\end{array}$ & $\begin{array}{c}0.056 \\
(1.53)\end{array}$ & $\begin{array}{l}0.025 * * \\
(2.68)\end{array}$ & $\begin{array}{l}0.040 * * \\
(3.31)\end{array}$ & $\begin{array}{l}0.041 \% \\
(2.53)\end{array}$ & $\begin{array}{c}0.027 \\
(1.38)\end{array}$ \\
\hline SSCI journal articles $(\ln ), t-1$ & $\begin{array}{l}-0.229 * * * \\
(-5.51)\end{array}$ & $\begin{array}{l}-0.204 * * * \\
(-5.34)\end{array}$ & $\begin{array}{l}-0.134 * * * \\
(-5.16)\end{array}$ & $\begin{array}{l}-0.099 * * * \\
(-4.96)\end{array}$ & $\begin{array}{l}-0.148^{* * *} \\
(-5.51)\end{array}$ & $\begin{array}{l}-0.142 * * * \\
(-6.16)\end{array}$ \\
\hline Books $(\ln ), t-1$ & $\begin{array}{c}0.040 \\
(0.91)\end{array}$ & $\begin{array}{c}0.002 \\
(0.06)\end{array}$ & $\begin{array}{c}0.018 \\
(0.67)\end{array}$ & $\begin{array}{l}-0.001 \\
(-0.04)\end{array}$ & $\begin{array}{c}0.028 \\
(1.00)\end{array}$ & $\begin{array}{c}0.009 \\
(0.39)\end{array}$ \\
\hline Book chapters $(\ln ), t-1$ & $\begin{array}{l}-0.013 \\
(-0.52)\end{array}$ & $\begin{array}{l}-0.063 * \\
(-2.11)\end{array}$ & $\begin{array}{l}-0.010 \\
(-0.71)\end{array}$ & $\begin{array}{l}-0.039 * * \\
(-2.92)\end{array}$ & $\begin{array}{l}-0.009 \\
(-0.57)\end{array}$ & $\begin{array}{l}-0.037^{*} \\
(-2.43)\end{array}$ \\
\hline Awards $(\ln ), t-1$ & $\begin{array}{c}0.076 \\
(0.49)\end{array}$ & $\begin{array}{c}0.104 \\
(1.09)\end{array}$ & $\begin{array}{r}0.095 \\
(1.60)\end{array}$ & $\begin{array}{c}0.067 \\
(1.24)\end{array}$ & $\begin{array}{c}0.058 \\
(0.78)\end{array}$ & $\begin{array}{c}0.066 \\
(1.10)\end{array}$ \\
\hline Co-authors $(\ln ), t-1$ & $\begin{array}{c}0.037 \\
(1.56)\end{array}$ & $\begin{array}{r}0.028 \\
(1.26)\end{array}$ & $\begin{array}{c}0.018 \\
(1.41)\end{array}$ & $\begin{array}{c}0.018 \\
(1.57)\end{array}$ & $\begin{array}{r}0.022 \\
(1.49)\end{array}$ & $\begin{array}{c}0.023^{+} \\
(1.72)\end{array}$ \\
\hline International publications $(\ln ), t-1$ & $\begin{array}{l}0.046^{+} \\
(1.65)\end{array}$ & $\begin{array}{l}0.078 * * \\
(2.99)\end{array}$ & $\begin{array}{l}0.033^{+} \\
(1.96)\end{array}$ & $\begin{array}{l}0.049 * * \\
(3.31)\end{array}$ & $\begin{array}{r}0.029 \\
(1.59)\end{array}$ & $\begin{array}{l}0.052 * * \\
(3.15)\end{array}$ \\
\hline DFG grants $(\ln ), t-1$ & $\begin{array}{c}0.082 \\
(1.34)\end{array}$ & $\begin{array}{c}0.233 \\
(1.14)\end{array}$ & $\begin{array}{c}0.057^{+} \\
(1.73)\end{array}$ & $\begin{array}{c}0.088 \\
(1.08)\end{array}$ & $\begin{array}{c}0.054 \\
(1.63)\end{array}$ & $\begin{array}{c}0.094 \\
(1.09)\end{array}$ \\
\hline Months abroad $(\ln ), t-1$ & $\begin{array}{l}0.036^{*} \\
(2.05)\end{array}$ & $\begin{array}{c}0.033 \\
(1.58)\end{array}$ & $\begin{array}{l}0.025 \% \\
(2.44)\end{array}$ & $\begin{array}{c}0.014 \\
(1.33)\end{array}$ & $\begin{array}{l}0.024 * \\
(2.17)\end{array}$ & $\begin{array}{r}0.015 \\
(1.30)\end{array}$ \\
\hline Mobility (ln), $t-1$ & $\begin{array}{c}-0.047 \\
(-1.28)\end{array}$ & $\begin{array}{c}0.077^{+} \\
(1.91)\end{array}$ & $\begin{array}{l}-0.022 \\
(-1.04)\end{array}$ & $\begin{array}{c}0.034 \\
(1.62)\end{array}$ & $\begin{array}{l}-0.028 \\
(-1.25)\end{array}$ & $\begin{array}{l}0.048 * \\
(1.97)\end{array}$ \\
\hline Interim professor $(\ln ), t-1$ & $\begin{array}{l}-0.057 \\
(-0.84)\end{array}$ & $\begin{array}{l}-0.110^{* *} \\
(-2.62)\end{array}$ & $\begin{array}{l}-0.051 \\
(-1.13)\end{array}$ & $\begin{array}{l}-0.060 \% \\
(-2.32)\end{array}$ & $\begin{array}{l}-0.042 \\
(-0.98)\end{array}$ & $\begin{array}{l}-0.065 \% \\
(-2.44)\end{array}$ \\
\hline Constant & $\begin{array}{l}0.087^{* * *} \\
(9.91)\end{array}$ & $\begin{array}{l}0.198 * * * \\
(57.17)\end{array}$ & $\begin{array}{l}0.064^{* * *} \\
(12.07)\end{array}$ & $\begin{array}{c}0.126^{* * * *} \\
(110.48)\end{array}$ & $\begin{array}{l}0.059 * * * \\
(10.15)\end{array}$ & $\begin{array}{l}0.128 * * * \\
(60.39)\end{array}$ \\
\hline$R^{2}$ overall & 0.000 & 0.000 & 0.006 & 0.007 & 0.000 & 0.002 \\
\hline$R^{2}$ within & 0.057 & 0.047 & 0.063 & 0.054 & 0.062 & 0.049 \\
\hline$R^{2}$ between & 0.020 & 0.027 & 0.001 & 0.006 & 0.016 & 0.070 \\
\hline$N$ (persons) & 282 & 411 & 297 & 432 & 282 & 411 \\
\hline$N$ (person-years) & 2,295 & 3,822 & 2,592 & 4,254 & 2,295 & 3,822 \\
\hline
\end{tabular}

Note: $t$ statistics in parentheses.

${ }^{+} P<0.1, * P<0.05, * * P<0.01, * * P<0.001$. 
Table A2. Replication of Table 3, Models 7 and 8, using fixed-effects individual slopes regression (dependent variable: annual number of SSCl journal articles for Models 1-4; log of annual no of SSCl journal articles for Models 5-8)

\begin{tabular}{|c|c|c|c|c|c|c|c|c|}
\hline & $\begin{array}{l}(1) \\
\text { Women } \\
\text { only }\end{array}$ & $\begin{array}{l}(2) \\
\text { Men } \\
\text { only }\end{array}$ & $\begin{array}{l}(3) \\
\text { Women } \\
\text { only }\end{array}$ & $\begin{array}{l}(4) \\
\text { Men } \\
\text { only }\end{array}$ & $\begin{array}{l}(5) \\
\text { Women } \\
\text { only }\end{array}$ & $\begin{array}{l}(6) \\
\text { Men } \\
\text { only }\end{array}$ & $\begin{array}{l}(7) \\
\text { Women } \\
\text { only }\end{array}$ & $\begin{array}{l}(8) \\
\text { Men } \\
\text { only }\end{array}$ \\
\hline Number of children $(\ln ), t-1$ & $\begin{array}{l}-0.092 * \\
(-2.08)\end{array}$ & $\begin{array}{l}-0.059 \\
(-1.14)\end{array}$ & & & $\begin{array}{l}-0.063 * \\
(-2.25)\end{array}$ & $\begin{array}{l}-0.030 \\
(-1.00)\end{array}$ & & \\
\hline Number of children $(\ln ), t-2$ & & & $\begin{array}{l}-0.121 * * \\
(-2.71)\end{array}$ & $\begin{array}{l}-0.020 \\
(-0.36)\end{array}$ & & & $\begin{array}{l}-0.081 * * \\
(-2.79)\end{array}$ & $\begin{array}{l}-0.008 \\
(-0.25)\end{array}$ \\
\hline Doctorate, $t-1$ & $\begin{array}{l}0.094 * * \\
(2.69)\end{array}$ & $\begin{array}{l}0.079 \% \\
(2.27)\end{array}$ & $\begin{array}{c}0.062^{+} \\
(1.81)\end{array}$ & $\begin{array}{l}0.083 * \\
(2.18)\end{array}$ & $\begin{array}{l}0.061 * * \\
(2.74)\end{array}$ & $\begin{array}{l}0.050 * \\
(2.48)\end{array}$ & $\begin{array}{l}0.045 * \\
(2.01)\end{array}$ & $\begin{array}{l}0.051 * \\
(2.37)\end{array}$ \\
\hline Habilitation, $t-1$ & $\begin{array}{l}-0.084^{+} \\
(-1.69)\end{array}$ & $\begin{array}{l}-0.074 \\
(-1.63)\end{array}$ & $\begin{array}{l}-0.091^{+} \\
(-1.69)\end{array}$ & $\begin{array}{l}-0.077^{+} \\
(-1.68)\end{array}$ & $\begin{array}{l}-0.051 \\
(-1.52)\end{array}$ & $\begin{array}{l}-0.049^{+} \\
(-1.92)\end{array}$ & $\begin{array}{l}-0.055 \\
(-1.51)\end{array}$ & $\begin{array}{l}-0.050^{+} \\
(-1.93)\end{array}$ \\
\hline Years in academia $(\ln ), t-1$ & $\begin{array}{l}0.045 * * \\
(3.08)\end{array}$ & $\begin{array}{l}0.105 * * * \\
(5.04)\end{array}$ & $\begin{array}{l}0.082 * * * \\
(3.56)\end{array}$ & $\begin{array}{l}0.125 * * * \\
(3.66)\end{array}$ & $\begin{array}{l}0.032 * * * \\
(3.34)\end{array}$ & $\begin{array}{l}0.068 * * * \\
(5.35)\end{array}$ & $\begin{array}{l}0.058^{* * * *} \\
(3.83)\end{array}$ & $\begin{array}{l}0.080 * * * \\
(3.84)\end{array}$ \\
\hline Books (ln), $t-1$ & $\begin{array}{l}-0.008 \\
(-0.20)\end{array}$ & $\begin{array}{c}0.012 \\
(0.33)\end{array}$ & $\begin{array}{l}-0.007 \\
(-0.14)\end{array}$ & $\begin{array}{c}0.012 \\
(0.30)\end{array}$ & $\begin{array}{l}-0.004 \\
(-0.17)\end{array}$ & $\begin{array}{c}0.012 \\
(0.53)\end{array}$ & $\begin{array}{l}-0.006 \\
(-0.21)\end{array}$ & $\begin{array}{r}0.015 \\
(0.61)\end{array}$ \\
\hline Book chapters $(\ln ), t-1$ & $\begin{array}{l}-0.006 \\
(-0.26)\end{array}$ & $\begin{array}{l}-0.053^{*} \\
(-2.26)\end{array}$ & $\begin{array}{c}0.002 \\
(0.09)\end{array}$ & $\begin{array}{l}-0.060 * \\
(-2.21)\end{array}$ & $\begin{array}{l}-0.007 \\
(-0.43)\end{array}$ & $\begin{array}{l}-0.037 * * \\
(-2.64)\end{array}$ & $\begin{array}{l}-0.002 \\
(-0.10)\end{array}$ & $\begin{array}{l}-0.042 * \\
(-2.58)\end{array}$ \\
\hline Co-authors $(\ln ), t-1$ & $\begin{array}{c}0.035^{+} \\
(1.73)\end{array}$ & $\begin{array}{c}0.030 \\
(1.33)\end{array}$ & $\begin{array}{c}0.028 \\
(1.15)\end{array}$ & $\begin{array}{c}0.033 \\
(1.32)\end{array}$ & $\begin{array}{c}0.023^{+} \\
(1.76)\end{array}$ & $\begin{array}{c}0.025^{+} \\
(1.81)\end{array}$ & $\begin{array}{c}0.017 \\
(1.10)\end{array}$ & $\begin{array}{l}0.027^{+} \\
(1.79)\end{array}$ \\
\hline International publications $(\ln ), t-1$ & $\begin{array}{l}0.102^{* *} \\
(3.20)\end{array}$ & $\begin{array}{l}0.094 * * * \\
(3.81)\end{array}$ & $\begin{array}{l}0.107^{* *} \\
(2.99)\end{array}$ & $\begin{array}{l}0.094 * * * \\
(3.64)\end{array}$ & $\begin{array}{l}0.067 * * \\
(3.15)\end{array}$ & $\begin{array}{l}0.061 * * * \\
(4.01)\end{array}$ & $\begin{array}{l}0.070 * * \\
(2.95)\end{array}$ & $\begin{array}{l}0.061 * * * \\
(3.81)\end{array}$ \\
\hline DFG grants $(\ln ), t-1$ & $\begin{array}{c}0.016 \\
(0.22)\end{array}$ & $\begin{array}{c}0.144 \\
(0.92)\end{array}$ & $\begin{array}{c}0.051 \\
(0.69)\end{array}$ & $\begin{array}{c}0.161 \\
(1.03)\end{array}$ & $\begin{array}{c}0.023 \\
(0.55)\end{array}$ & $\begin{array}{l}0.075 \\
(0.96)\end{array}$ & $\begin{array}{c}0.046 \\
(1.04)\end{array}$ & $\begin{array}{c}0.092 \\
(1.12)\end{array}$ \\
\hline Months abroad $(\ln ), t-1$ & $\begin{array}{c}0.016 \\
(1.03)\end{array}$ & $\begin{array}{l}0.006 \\
(0.34)\end{array}$ & $\begin{array}{c}0.018 \\
(0.98)\end{array}$ & $\begin{array}{c}0.009 \\
(0.48)\end{array}$ & $\begin{array}{r}0.010 \\
(0.99)\end{array}$ & $\begin{array}{c}0.001 \\
(0.12)\end{array}$ & $\begin{array}{c}0.010 \\
(0.89)\end{array}$ & $\begin{array}{c}0.004 \\
(0.34)\end{array}$ \\
\hline Mobility $(\ln ), t-1$ & $\begin{array}{l}-0.042 \\
(-1.15)\end{array}$ & $\begin{array}{c}0.044 \\
(1.07)\end{array}$ & $\begin{array}{l}-0.042 \\
(-1.01)\end{array}$ & $\begin{array}{c}0.052 \\
(1.13)\end{array}$ & $\begin{array}{l}-0.022 \\
(-0.97)\end{array}$ & $\begin{array}{c}0.024 \\
(0.98)\end{array}$ & $\begin{array}{l}-0.021 \\
(-0.82)\end{array}$ & $\begin{array}{c}0.029 \\
(1.05)\end{array}$ \\
\hline Interim professor $(\ln ), t-1$ & $\begin{array}{l}-0.027 \\
(-0.42)\end{array}$ & $\begin{array}{l}-0.084^{+} \\
(-1.72)\end{array}$ & $\begin{array}{l}-0.022 \\
(-0.29)\end{array}$ & $\begin{array}{l}-0.087^{+} \\
(-1.79)\end{array}$ & $\begin{array}{l}-0.021 \\
(-0.51)\end{array}$ & $\begin{array}{l}-0.043 \\
(-1.48)\end{array}$ & $\begin{array}{l}-0.017 \\
(-0.35)\end{array}$ & $\begin{array}{l}-0.045 \\
(-1.52)\end{array}$ \\
\hline$R^{2}$ within & 0.062 & 0.054 & 0.064 & 0.051 & 0.069 & 0.066 & 0.072 & 0.063 \\
\hline$N$ (persons) & 233 & 347 & 212 & 315 & 233 & 347 & 212 & 315 \\
\hline$N$ (person-years) & 2,451 & 4,070 & 2,155 & 3,627 & 2,451 & 4,070 & 2,155 & 3,627 \\
\hline
\end{tabular}

Note: $t$ statistics in parentheses.

${ }^{+} P<0.1, " P<0.05, * * P<0.01, * * * P<0.001$. 
Table A3. Replication of Table 3 using dummy variables for number of children (dependent variable: annual number of $\mathrm{SSCl}$ journal articles)

\begin{tabular}{|c|c|c|c|c|c|c|c|c|}
\hline & $\begin{array}{l}(1) \\
\text { Women } \\
\text { only }\end{array}$ & $\begin{array}{l}(2) \\
\text { Men } \\
\text { only }\end{array}$ & $\begin{array}{l}(3) \\
\text { Women } \\
\text { only }\end{array}$ & $\begin{array}{l}(4) \\
\text { Men } \\
\text { only }\end{array}$ & $\begin{array}{l}(5) \\
\text { Women } \\
\text { only }\end{array}$ & $\begin{array}{l}\text { (6) } \\
\text { Men } \\
\text { only }\end{array}$ & $\begin{array}{l}(7) \\
\text { Women } \\
\text { only }\end{array}$ & $\begin{array}{l}(8) \\
\text { Men } \\
\text { only }\end{array}$ \\
\hline Number of children $=1, t-2$ & $\begin{array}{c}0.039 \\
(1.38)\end{array}$ & $\begin{array}{l}0.091 * \\
(2.44)\end{array}$ & $\begin{array}{l}-0.053 \\
(-1.61)\end{array}$ & $\begin{array}{c}0.022 \\
(0.52)\end{array}$ & $\begin{array}{l}-0.069 * \\
(-2.02)\end{array}$ & $\begin{array}{c}0.034 \\
(0.73)\end{array}$ & $\begin{array}{l}-0.068 * \\
(-1.99)\end{array}$ & $\begin{array}{c}0.032 \\
(0.69)\end{array}$ \\
\hline Number of children $=2, t-2$ & $\begin{array}{l}0.020 \\
(0.65)\end{array}$ & $\begin{array}{l}0.116 \% \\
(2.42)\end{array}$ & $\begin{array}{l}-0.108 * * \\
(-2.66)\end{array}$ & $\begin{array}{c}0.012 \\
(0.22)\end{array}$ & $\begin{array}{l}-0.129 * * \\
(-2.68)\end{array}$ & $\begin{array}{l}0.016 \\
(0.25)\end{array}$ & $\begin{array}{l}-0.126^{*} \\
(-2.53)\end{array}$ & $\begin{array}{c}0.014 \\
(0.21)\end{array}$ \\
\hline Number of children $\geq 3, t-2$ & $\begin{array}{c}0.129 \\
(0.99)\end{array}$ & $\begin{array}{l}0.112 * \\
(2.30)\end{array}$ & $\begin{array}{l}-0.070 \\
(-0.50)\end{array}$ & $\begin{array}{l}-0.032 \\
(-0.51)\end{array}$ & $\begin{array}{l}-0.065 \\
(-0.46)\end{array}$ & $\begin{array}{c}-0.017 \\
(-0.23)\end{array}$ & $\begin{array}{l}-0.045 \\
(-0.32)\end{array}$ & $\begin{array}{l}-0.024 \\
(-0.33)\end{array}$ \\
\hline Doctorate, $t-1$ & & & $\begin{array}{l}0.086^{* *} \\
(3.12)\end{array}$ & $\begin{array}{l}0.108 * * \\
(3.26)\end{array}$ & $\begin{array}{l}0.090 * \\
(2.56)\end{array}$ & $\begin{array}{l}0.115^{* *} \\
(3.23)\end{array}$ & $\begin{array}{l}0.089 * \\
(2.56)\end{array}$ & $\begin{array}{l}0.098 * * \\
(2.75)\end{array}$ \\
\hline Habilitation, $t-1$ & & & $\begin{array}{l}-0.065 \\
(-1.57)\end{array}$ & $\begin{array}{l}-0.061 \\
(-1.36)\end{array}$ & $\begin{array}{l}-0.053 \\
(-1.13)\end{array}$ & $\begin{array}{l}-0.030 \\
(-0.62)\end{array}$ & $\begin{array}{l}-0.043 \\
(-0.83)\end{array}$ & $\begin{array}{l}-0.012 \\
(-0.25)\end{array}$ \\
\hline Years in academia $(\ln ), t-1$ & & & $\begin{array}{l}0.061 * * \\
(3.17)\end{array}$ & $\begin{array}{l}0.041 \% \\
(2.43)\end{array}$ & $\begin{array}{l}0.064 \% \\
(2.51)\end{array}$ & $\begin{array}{c}0.068^{+} \\
(1.86)\end{array}$ & $\begin{array}{l}0.059 * \\
(2.32)\end{array}$ & $\begin{array}{l}0.055 \\
(1.50)\end{array}$ \\
\hline SSCI journal articles $(\ln ), t-1$ & & & & & $\begin{array}{l}-0.229 * * \\
(-5.52)\end{array}$ & $\begin{array}{l}-0.209 * * * \\
(-5.55)\end{array}$ & $\begin{array}{l}-0.231 * * * \\
(-5.56)\end{array}$ & $\begin{array}{l}-0.205 * * * \\
(-5.37)\end{array}$ \\
\hline Books $(\ln ), t-1$ & & & & & $\begin{array}{l}0.041 \\
(0.94)\end{array}$ & $\begin{array}{l}-0.005 \\
(-0.12)\end{array}$ & $\begin{array}{c}0.038 \\
(0.87)\end{array}$ & $\begin{array}{r}0.001 \\
(0.03)\end{array}$ \\
\hline Book chapters $(\ln ), t-1$ & & & & & $\begin{array}{l}-0.021 \\
(-0.80)\end{array}$ & $\begin{array}{l}-0.054^{+} \\
(-1.83)\end{array}$ & $\begin{array}{l}-0.015 \\
(-0.59)\end{array}$ & $\begin{array}{l}-0.060 * \\
(-2.03)\end{array}$ \\
\hline Awards $(\ln ), t-1$ & & & & & $\begin{array}{c}0.091 \\
(0.60)\end{array}$ & $\begin{array}{c}0.109 \\
(1.17)\end{array}$ & $\begin{array}{c}0.080 \\
(0.52)\end{array}$ & $\begin{array}{c}0.108 \\
(1.13)\end{array}$ \\
\hline Co-authors (ln), $t-1$ & & & & & $\begin{array}{c}0.033 \\
(1.40)\end{array}$ & $\begin{array}{c}0.028 \\
(1.21)\end{array}$ & $\begin{array}{c}0.037 \\
(1.56)\end{array}$ & $\begin{array}{r}0.027 \\
(1.21)\end{array}$ \\
\hline International publications $(\ln ), t-1$ & & & & & $\begin{array}{l}0.056 * \\
(2.04)\end{array}$ & $\begin{array}{l}0.090 * * * \\
(3.33)\end{array}$ & $\begin{array}{c}0.044 \\
(1.61)\end{array}$ & $\begin{array}{l}0.077 * * \\
(2.99)\end{array}$ \\
\hline DFG grants $(\ln ), t-1$ & & & & & $\begin{array}{c}0.067 \\
(1.12)\end{array}$ & $\begin{array}{c}0.237 \\
(1.13)\end{array}$ & $\begin{array}{r}0.086 \\
(1.41)\end{array}$ & $\begin{array}{c}0.236 \\
(1.15)\end{array}$ \\
\hline Months abroad $(\ln ), t-1$ & & & & & & & $\begin{array}{l}0.037^{*} \\
(2.11)\end{array}$ & $\begin{array}{c}0.033 \\
(1.58)\end{array}$ \\
\hline Mobility $(\ln ), t-1$ & & & & & & & $\begin{array}{l}-0.047 \\
(-1.29)\end{array}$ & $\begin{array}{c}0.078^{+} \\
(1.94)\end{array}$ \\
\hline Interim professor $(\ln ), t-1$ & & & & & & & $\begin{array}{l}-0.054 \\
(-0.79)\end{array}$ & $\begin{array}{l}-0.108^{* *} \\
(-2.59)\end{array}$ \\
\hline Constant & $\begin{array}{l}0.128 * * * \\
(20.47)\end{array}$ & $\begin{array}{l}0.170 * * * \\
(12.13)\end{array}$ & $\begin{array}{l}0.152 * * * \\
(16.82)\end{array}$ & $\begin{array}{l}0.194 * * * \\
(11.81)\end{array}$ & $\begin{array}{l}0.124 * * * \\
(9.30)\end{array}$ & $\begin{array}{l}0.189 * * * \\
(10.42)\end{array}$ & $\begin{array}{l}0.118 * * * \\
(8.83)\end{array}$ & $\begin{array}{l}0.191 * * * \\
(10.58)\end{array}$ \\
\hline$R^{2}$ overall & 0.000 & 0.000 & 0.021 & 0.012 & 0.003 & 0.000 & 0.000 & 0.000 \\
\hline$R^{2}$ within & 0.001 & 0.005 & 0.026 & 0.018 & 0.053 & 0.043 & 0.058 & 0.047 \\
\hline$R^{2}$ between & 0.001 & 0.002 & 0.036 & 0.028 & 0.077 & 0.056 & 0.021 & 0.027 \\
\hline$N$ (persons) & 282 & 411 & 282 & 411 & 282 & 411 & 282 & 411 \\
\hline$N$ (person-years) & 2,295 & 3,822 & 2,295 & 3,822 & 2,295 & 3,822 & 2,295 & 3,822 \\
\hline
\end{tabular}

Note: $t$ statistics in parentheses.

${ }^{+} P<0.1,{ }^{*} P<0.05, * * P<0.01, * * * P<0.001$. 